\title{
The encephalomyocarditis virus Leader promotes the release of virions inside extracellular vesicles via the induction of secretory autophagy
}

Susanne G. van der Grein ${ }^{1,4}$, Kyra A.Y. Defourny ${ }^{1,4}$, Huib H. Rabouw ${ }^{2}$, Soenita S. Goerdayal' ${ }^{3}$, Martijn J.C. van Herwijnen ${ }^{1}$, Richard W. Wubbolts ${ }^{1}$, Maarten Altelaar ${ }^{3}$, Frank J.M. van Kuppeveld ${ }^{2}$, Esther N.M. Nolte-'t Hoen ${ }^{1 *}$.

${ }^{1}$ Division of Cell Biology, Metabolism \& Cancer, Department of Biomolecular Health Sciences, Faculty of Veterinary Medicine, Utrecht University, Yalelaan 2, 3584 CM Utrecht, The Netherlands.

${ }^{2}$ Virology Section, Division Infectious Diseases \& Immunology, Department of Biomolecular Health Sciences, Faculty of Veterinary Medicine, Utrecht University, Yalelaan 1, 3584 CL Utrecht, The Netherlands.

${ }^{3}$ Biomolecular Mass Spectrometry and Proteomics, Bijvoet Center for Biomolecular Research and Utrecht Institute for Pharmaceutical Sciences, Utrecht University, Padualaan 8, 3584 CH Utrecht, The Netherlands.

${ }^{4}$ These authors contributed equally

*Correspondence should be addressed to:

e.n.m.nolte@uu.nl

\section{Keywords}

Extracellular vesicles, exosomes, secretory autophagy, picornavirus, non-lytic virus release, viral security proteins

\section{Summary}

Naked viruses can escape host cells before the induction of lysis via release in extracellular vesicles (EVs). These nanosized EVs cloak the secreted virus particles in a host-derived membrane, which alters virus-host interactions that affect infection efficiency and antiviral immunity. Currently, little is known about the viral and host factors regulating this form of virus release. Here, we assessed the role of picornavirus security proteins by studying the encephalomyocarditis virus (EMCV) Leader protein. EV release upon infection with wildtype virus or a Leader-deficient mutant was characterized at the single particle level using high-resolution flow cytometry. Inactivation of the Leader abolished $\mathrm{EV}$ induction during infection and strongly reduced $\mathrm{EV}$-enclosed virus release. We demonstrate that the Leader promotes the release of virions within EVs by stimulating a secretory arm of autophagy. This newly discovered role of the EMCV Leader adds to the variety of mechanisms via which this protein affects virus-host interactions. Moreover, these data provide first evidence for a crucial role of non-structural viral proteins in the non-lytic release of picornaviruses via packaging in EVs.

\section{Introduction}

Picornaviruses form a large family of small $(\sim 30 \mathrm{~nm})$ single-stranded positive-sense RNA viruses that have long been believed to be exclusively released from cells as non-enveloped or 'naked' progeny by inducing cell rupture. However, we and others showed that several picornavirus species, including hepatitis A virus (HAV), poliovirus, enterovirus 71 (EV71), coxsackievirus B3 (CVB3), and 
encephalomyocarditis virus (EMCV), can also be released from cells in a non-lytic fashion via packaging in extracellular vesicles $(E V s)^{1-5}$. EVs are nanosized $(50-300 \mathrm{~nm})$ lipid bilayer-enclosed particles that are released by all types of cells and deliver protein or RNA cargo to distant or neighbouring cells as a means of intercellular communication (reviewed in ${ }^{6,7}$ ). The existence of EVenclosed virus particles in vivo has been confirmed for several types of naked viruses, including picornaviruses, hepatitis E virus, rotavirus, and norovirus ${ }^{1,4,8-10}$, and raises important questions regarding their role in infection. It has been well established that virus-containing EVs can transfer the infection to new host cells. Importantly, EV-enclosed viruses may differ in biological properties compared to their naked counterparts. Incorporation in EVs allows viruses to escape immune surveillance as the EV membrane shields virions from recognition by neutralizing antibodies ${ }^{1,11-13}$. Likewise, the EV membrane may alter cellular uptake dynamics and affect biodistribution of the virus, including passage over barriers such as the blood-brain barrier ${ }^{14-19}$. In addition, EV-enclosure may increase replicative fitness of the virus through simultaneous delivery of multiple genetic quasispecies ${ }^{3,10,20}$. As a result, EV-enclosure of viruses can increase infection efficiency. Conversely, the transfer of virus or host molecules from infected cells via EVs may also trigger antiviral immune responses in targeted cells, thereby restricting the spread of the infection ${ }^{21-23}$.

While several lines of evidence indicate the functional importance of EV-enclosed virus release, there are many unknowns regarding the formation of virus-containing EVs. The most common EV release pathways are the outward pinching of vesicles from the plasma membrane and the release of intraluminal vesicles via fusion of multivesicular endosomes (MVE) with the plasma membrane (reviewed in ${ }^{6}$ ). Recently, several picornavirus species were reported to escape cells enwrapped in EVs containing the autophagy-regulatory protein $\mathrm{LC} 3^{2,3,5,24,25}$. This finding triggered the idea that also autophagy could be involved in formation of EV-enclosed viruses. Autophagy is a cellular process in which damaged or obsolete organelles, aggregated proteins, or intracellular pathogens are engulfed and broken down to recycle nutrients and maintain homeostasis ${ }^{26}$. While autophagy is often employed by host cells to defend themselves against invading viruses, several picornaviruses actively induce autophagy to support virus replication and release ${ }^{27,28}$. Based on the reported presence of LC3 on virus-containing EVs, it has been proposed that instead of following conventional routing towards lysosomes, autophagosomes that have engulfed nascent virions may expel their contents to the external milieu during viral infection. The process in which autophagosomal cargo is secreted into the extracellular environment instead of being degraded is generally referred to as 'secretory autophagy'. This process is known to facilitate unconventional secretion of cytosolic proteins lacking a signal peptide for the classical secretory pathway ${ }^{29}$. Although a role for autophagy in non-lytic virus release has been suggested, direct evidence for the specific involvement of a secretory arm of the autophagy pathway in the biogenesis and release of virus-containing EVs is currently lacking. In addition, it is unknown which viral and/or host factors are involved in regulating this form of virus release.

Here, we investigated whether and how viral proteins actively promote the release of EV-enclosed viruses by studying the Leader protein of encephalomyocarditis virus (EMCV), a model picornavirus with a rapid lytic life cycle. The EMCV Leader functions in the subversion of antiviral defense as well as the manipulation of host transcription and translation, and is therefore referred to as the main 'viral security protein' of EMCV ${ }^{30}$. Previously, we demonstrated that EMCV induces the release of multiple EV populations and that only specific EV subpopulations were highly potent in spreading the infection $^{5}$. We here investigated whether the EMCV Leader protein plays a role in the release of these infectious EVs using high-resolution flow cytometric quantification and characterization of single EVs. We demonstrate that the Leader protein acts as a major determinant for the release of 
virus-containing EV subpopulations. By tracking the fate of the autophagic marker LC3, we show that the Leader promotes the accumulation of autophagic structures that do not progress along the degradation pathway and that the Leader is required for the induction of secretory autophagy during infection. Pharmacological treatments validate that via the activation of secretory autophagy the Leader protein promotes the packaging of virus particles within EVs. These data exemplify the importance of non-structural viral proteins in promoting the release of EV-enclosed virions via modification of host cell secretory pathways.

\section{Results}

\section{Inactivation of the Leader protein reduces EV-enclosed virus release by EMCV-infected cells}

The EMCV Leader is of critical importance for in vivo infection, yet was previously shown to be dispensable for virus replication ${ }^{31}$. Here, we set out to study the role of the Leader protein in the regulation of EV-enclosed virus release. Non-lytic release of virus and EVs was assessed in cells infected with either wildtype (Wt) EMCV or with a previously established mutant (EMCV-L $\mathrm{L}^{\mathrm{Zn}}$ ) expressing a functionally inactive Leader protein ${ }^{31}$. Similar replication kinetics were observed for both viruses during the pre-lytic phase of a single round of infection at a high multiplicity of infection (MOI 10) (Figure 1A). Whereas only minor differences in total virus production were observed, EMCV- $\mathrm{L}^{\mathrm{Zn}}$ infected cells released substantially lower amounts of virus into the extracellular space than EMCVWt infected cells upon reaching a plateau in virus production (7-8 hrs p.i.) (Figure 1B). Prolonged culture past $8 \mathrm{hrs}$ p.i. led to virus-induced cell lysis for EMCV-Wt infected cells, whereas EMCV- $\mathrm{L}^{2 n}$ infected cells could be cultured without loss of cell viability up to $12 \mathrm{hrs}$ p.i. However, virus release by EMCV- $\mathrm{L}^{\mathrm{Zn}}$ infected cells at this later time point did not reach the level of virus release observed for EMCV-Wt at $8 \mathrm{hrs}$ p.i. (Figure 1B, Supplementary figure 1A). Virus packaging in EVs is believed to be the main form of virus release in the early or pre-lytic stages of the infection. We therefore investigated whether the defect in extracellular virus release up to $8 \mathrm{hrs}$ p.i. was indicative of a defect in EV-enclosed virus release. We previously reported that EMCV-Wt infected cells release virus within different $E V$ subsets that can be isolated from cell culture supernatant by centrifugation at either $10,000 \times \mathrm{x}(10 \mathrm{~K})$ or $100,000 \times \mathrm{xg}(100 \mathrm{~K})$ centrifugal force ${ }^{5}$. In contrast to EMCV-Wt infected cells, little to no $10 \mathrm{~K}$ EVs were found to be released by EMCV- $\mathrm{L}^{2 \mathrm{n}}$ infected cells (data not shown). We therefore focused on comparing the amount of virus incorporated in the $100 \mathrm{~K}$ EVs released during EMCV-Wt and EMCV-L $\mathrm{L}^{\mathrm{Zn}}$ infection. 100K EVs and EV-enclosed virus particles were separated from potentially co-isolating naked viruses based on buoyant density by means of isopycnic density gradient centrifugation ${ }^{5}$. Significantly more infectivity was detected in the EV isolates from EMCV-Wt compared to EMCV- $\mathrm{L}^{\mathrm{Zn}}$ infected samples (Figure 1C), a difference that could not be explained by differences in intracellular virus production (Supplementary figure 1B). To validate that this decrease in infectivity was due to a lack of virus incorporation into EV, we performed LC-MS/MS analysis of the EV samples and mapped the resulting peptide hits against the EMCV polyprotein. Seventeen different unique viral peptides were identified across the three replicate preparations of EMCV-Wt EVs (Figure 1D). Most (13/17) unique peptides were identified within the capsid coding region of the EMCV polyprotein, which supports the notion that EVs released by EMCV-Wt infected cells incorporate mature virions. In contrast, no viral peptides were detected in EMCV- $\mathrm{L}^{\mathrm{Zn}}$ induced EVs. Hence, the presence of the Leader strongly promotes the packaging and/or release of EV-enclosed EMCV. 


\section{The EMCV Leader promotes the release of specific virus-containing EV subsets}

The reduction in EV-enclosed virus release by cells infected with the Leader-deficient virus prompted us to further explore the role of the Leader in the induction of EV release. To this end, we analyzed EVs harvested at different time points post infection using an in-house developed, high-resolution flow cytometry-based approach for the quantification and characterization of $\mathrm{EVs}^{32,33}$. Using this approach, we observed that EMCV-Wt infection caused a sharp increase in the overall number of $100 \mathrm{~K}$ EVs released from $6 \mathrm{hrs}$ p.i. onwards (Figure 2A). However, in cells infected with EMCV-L ${ }^{2 n}$, this virus-induced increase in $\mathrm{EV}$ release was completely abolished, resulting in a significantly lower number of released EVs at 8 hrs p.i. for EMCV- $\mathrm{L}^{\mathrm{Zn}}$ infected cells compared to EMCV-Wt infected cells (Figure 2A, B). Closer examination of the flow cytometry dot plots revealed an additional phenotypic difference between the released EVs. EMCV-Wt induced the release of two EV subpopulations with different forward scattered light (FSC) intensities (Figure 2C), of which the population of FSC-high EVs is most efficient in spreading infection to other cells ${ }^{5}$. Over the course of the infection, an increase in the percentage of FSC-high EVs released by cells infected with EMCV-Wt was observed (Figure 2D, E). In contrast, the percentage of FSC-high EVs released from cells infected with EMCV-L ${ }^{\mathrm{Zn}}$ remained comparable to mock cells throughout the infection. These results indicate that the EMCV Leader protein promotes the release of a specific subset of FSC-high EVs that were previously shown to efficiently contribute to EMCV transmission.

\section{The EV proteome suggests a potential role for secretory autophagy in Leader-induced EV release}

To further investigate how the Leader protein affects the composition and release of EVs and EVenclosed virus, we compared the host protein composition of EV preparations ( $n=3$ replicates) from cells infected with EMCV-Wt or EMCV- $\mathrm{L}^{\mathrm{Zn}}$. Proteins involved in $\mathrm{EV}$ formation and release have frequently been identified as integral components of EVs. Comparative analysis of EV proteomes can therefore provide valuable information on how the Leader protein affects pathways involved in $\mathrm{EV}$ formation and release. Of the total number of proteins identified in EVs from infected cells by LC$\mathrm{MS} / \mathrm{MS}$ ( $\mathrm{N}=234$ for EMCV-Wt, and N=275 for $E M C V-\mathrm{L}^{\mathrm{Zn}}$ ), 190 proteins were detected for both viruses, while 44 proteins were exclusively identified in EMCV-Wt-induced EVs (Figure 3A, Supplementary table 1). The 44 proteins that were dependent on the Leader for incorporation into EVs were subjected to functional enrichment analysis. In the Gene Ontology (GO) domain 'cellular component', these proteins were highly enriched ( $>10$ fold enrichment $v s$. genome) for ER-Golgi intermediate compartment (ERGIC) membrane, and secretory granule membrane components, as well as components of ER-to-Golgi transport vesicles (Figure 3B). Subsequent analysis of protein interaction networks revealed an interconnected group of proteins in EMCV-Wt EV that were linked to these enriched GO terms (Figure 3C). Furthermore, GO analysis for 'biological process' revealed highly enriched terms associated with vesicular transport between ER and Golgi, as well as post-Golgi vesicle mediated transport (Figure 3D). Protein-network analysis revealed the involvement of a core group of interacting proteins, which included regulators of trafficking between ER and Golgi (TFG, TMED9, TMED10), proteins implicated in trafficking between Golgi and endosomes (Rab14, LMAN2), and SNARE proteins (Sec22b, SNAP23, STX4) (Figure 3E). The plasma membrane-resident SNARE proteins SNAP23 and STX4 were recently implicated in EV release by regulating the fusion of MVBs with the plasma membrane ${ }^{34,35}$. Moreover, by interacting with Sec22b upon its recruitment to autophagosomal membranes, this combination of SNARE proteins was also shown to facilitate a secretory arm of the autophagy pathway that enables the release of the pro-inflammatory cytokine IL- $1 \beta^{36}$. Both ERGIC and Golgi compartments are considered membrane sources for autophagosome formation ${ }^{37-39}$. Enrichment of GO-terms related to these compartments in EMCV-Wt-EVs could 
support the notion that autophagosomes intersect with the site of EV biogenesis.

The intersection of autophagosomes with compartments involved in EV biogenesis can result in the extracellular release of autophagic cargo. We therefore expanded our analysis of the secretome of infected cells by western blotting for the autophagy marker LC3 in the crude $100 \mathrm{~K}$ pellets. We observed that the 100K EV pellets from EMCV-Wt infected cells contained LC3, whereas there was no notable release of LC3 from cells infected with EMCV-L ${ }^{2 n}$ (Figure 3F). In contrast, the release of the typical EV protein CD63 was unchanged in the absence of a functional Leader protein. These data support a role of the EMCV Leader in the activation of a secretory arm of the autophagy pathway. The majority of the LC3 released by EMCV-Wt-infected cells was sensitive to treatment with proteinase K (Figure 3G), indicating that this LC3 was not enclosed within the EV lumen, but instead was secreted alongside or associated to EVs. However, part of the LC3 signal, likely corresponding to the lipidated form of LC3 (LC3-II), was protected from protease digestion in the absence of detergents indicating that LC3 is also partially incorporated in EMCV-Wt induced EVs. This Leaderdependent secretion of LC3, combined with the presence of Sec22b, SNAP23 and STX4 as well as Golgi/ERGIC components in EVs from EMCV-infected cells hints at a role for secretory autophagy in the release of virus-containing EVs.

\section{The EMCV Leader modulates autophagic flux during infection}

To further investigate the role of the EMCV Leader in the activation of secretory autophagy, we first assessed whether the Leader affected the overall level of autophagy in infected cells. Immunoblot analysis of EMCV-Wt and $-\mathrm{L}^{\mathrm{Zn}}$ infected cells showed that both viruses promoted the conversion of LC3I to its phosphatidylethanolamine-conjugated form (LC3II), indicating an increase in the number of autophagosomes compared to uninfected cells (Figure 4A). However, this increase in LC3II/I ratios was more pronounced in cells infected with EMCV-Wt. In addition, we noted that infection with EMCV-Wt, but not EMCV-L ${ }^{\mathrm{Zn}}$, consistently increased the presence of an additional, intermediate LC3 band. This banding pattern has previously been ascribed to a pro-form of LC3, although the migration patterns of LC3 forms remain a matter of debate ${ }^{40-42}$. We next assessed whether the Leader-induced increase in LC3II-containing autophagosomes was the result of a defect in the degradation of autophagosomes by fusion to lysosomes. Hereto, we made use of cells expressing tandemfluorochrome tagged LC3 (mCherry-EGFP-LC3) in which autophagosomes can be distinguished from autolysosomes based on their EGFP:mCherry fluorescence ratio (Figure 4B). In this reporter system, the drop in $\mathrm{pH}$ caused by the fusion of autophagosomes with late endosomes or lysosomes causes the loss of LC3-EGFP fluorescence in autolysosomes while the more acid-resistant mCherry fluorescence persists. Using confocal imaging, we detected an increase in the relative number of autophagosomes $\left(\mathrm{EGFP}^{+} \mathrm{mCherry}{ }^{+}\right.$puncta) versus autolysosomes (EGFP-mCherry ${ }^{+}$puncta) over the course of EMCV-Wt infection (Figure $4 \mathrm{C}$ ). In contrast, no differences were observed in the ratio between these two compartments in EMCV- $\mathrm{L}^{\mathrm{Zn}}$ infected cells compared to mock treated controls, although an increase in LC3II levels was also observed upon EMCV-L ${ }^{2 n}$ infection. We investigated whether the accumulation of autophagosomes was caused by a Leader-induced defect in the lysosomal system, and hence an inability to form acidified autolysosomes, by tracing the intensities of lysotracker stained cells. Lysotracker staining of acidified (endo)lysosomal compartments was similar between cells infected with EMCV-Wt and EMCV- $\mathrm{L}^{\mathrm{Zn}}$ (Figure 4D). Thus, the Leader induces an accumulation of autophagosomes without causing defects in lysosomal function.

Next, we determined whether the observed reduction in autophagosome turnover in the presence of the Leader contributed to the induction of secretory autophagy and the release of EV-enclosed virus. 
To this end, we tested the impact of rapamycin treatment on EMCV-Wt infected cells. Rapamycin is an inhibitor of mTOR and a well-known stimulator of autophagic flux. Treatment with rapamycin partially restored the balance in the ratio of autophagosomes vs. autolysosomes without affecting virus replication or cell viability (Figure 5A, Supplementary figure 2A, B), indicating that infected cells were still capable of autolysosome formation. Despite an increase in autophagic degradation, we observed no concomitant reduction in the release of LC3 upon rapamycin treatment, indicating that the level of secretory autophagy was not diminished (Figure 5B). Moreover, rapamycin had no effect on the overall amount of EV-enclosed virus released by infected cells (Figure $\mathbf{5 C}$ ), although the altered cellular condition induced by this drug caused the virus to be preferentially packaged in $10 \mathrm{~K}$ rather than 100K EVs (Figure 5D). These data suggest that Leader-induced secretion of autophagosome cargo and EV-enclosed viruses is not caused by defective or insufficient (auto)lysosomal degradation. Instead, we propose that the Leader specifically induces the activation of a secretory arm of the autophagy pathway.

\section{The induction of secretory autophagy promotes EV-enclosed virus release}

Finally, we investigated whether the induction of secretory autophagy and the packaging of EMCV virions in EVs were causally linked. If that were the case, we reasoned that pharmacological induction of secretory autophagy might rescue the defect in EV-enclosed virus release caused by mutation of the EMCV Leader. To test this hypothesis, we treated cells with apilimod prior to infection with EMCV- $\mathrm{L}^{\mathrm{Zn}}$. Apilimod prevents lysosome regeneration and prolonged exposure of cells was previously shown to trigger the extracellular release of autophagosome cargo ${ }^{43}$. To verify that the effects induced by apilimod were due to the specific induction of secretory autophagy rather than the inhibition of lysosome function during the course of the infection, we treated cells with bafilomycin as a negative control. Bafilomycin functions as an inhibitor of lysosomal degradation, similar to apilimod, by preventing lysosomal acidification (Figure 4D). Under our treatment regimes, neither drug negatively affected cell viability (Supplementary figure 4). Apilimod, but not bafilomycin, efficiently induced secretory autophagy in EMCV-L ${ }^{\mathrm{Zn}}$ infected cells, indicated by the robust presence of LC3 in 100K EV pellets (Figure 6A). However, both drugs induced a similar increase in total EV release (Figure 6B). Importantly, apilimod treatment (partially) rescued EV-enclosed virus release by EMCV- $\mathrm{L}^{\mathrm{Zn}}$ infected cells (Figure $6 \mathrm{C}$ ), despite causing an overall decrease in intracellular virus production (Figure 6D). In contrast, bafilomycin treatment did not promote the capacity of EMCV-L $\mathrm{L}^{2 \mathrm{n}}$ infected cells to release EV-enclosed virus. These findings indicate that the release of viruses in EVs is not merely controlled by the overall amount of EV production by infected cells. Instead, virus packaging in EVs could be specifically enhanced by the pharmacological induction of secretory autophagy, a process that is enabled by the viral Leader protein during EMCV infection.

\section{Discussion}

The data presented here highlights the first example of a non-structural viral protein regulating the release of EV-enclosed viruses. We showed that the EMCV Leader, a viral security protein, was crucial in triggering the release of EV-enclosed progeny virus from EMCV-infected cells. We demonstrated that the Leader triggers the release of a specific subset of EVs previously demonstrated to efficiently transmit EMCV infection ${ }^{5}$. In contrast, EV release by cells infected with a Leader-deficient virus was comparable to mock-infected cells, and these constitutively released EVs failed to efficiently package virus particles. Finally, we demonstrate a role for secretory autophagy in the Leader-mediated induction of EV-enclosed virus release. 
Our findings represent a novel addition to the variety of functions ascribed to the EMCV Leader and shed new light on the role of this protein in disease. The Leader is of critical importance for virus pathogenesis in vivo, and its absence strongly reduces morbidity and mortality in mice ${ }^{31}$. The contribution of the Cardiovirus Leader to efficient virus replication and spreading has been ascribed to its ability to interfere with the induction of antiviral defense pathways, such as the type I IFN response, the cellular stress response, and the induction of apoptotic cell death ${ }^{30,31,44,45}$. By promoting the release of EV-enclosed viruses, the Leader additionally enables the formation of infectious particles that are functionally distinct from naked virions, both in their uptake dynamics and in their accessibility to the humoral immune system due to the surrounding EV membrane $e^{1,3,10,13,18,19,46}$. As a result, the formation of EV-enclosed virus represents an additional mechanism via which the Leader may enable immune escape and efficient virus spread during EMCV infection.

Our data indicate that the Leader induces the packaging and release of viruses in specific EV subpopulations and that the secretory arm of autophagy plays a role in the formation of these viruscarrying EVs. Autophagy was previously shown to be required for the unconventional secretion of cytosolic proteins that lack a signal peptide. Importantly, many of the proteins shown to depend on autophagy for extracellular release were also found to be packaged in EVs. The cytokine IL1 $\beta$, a prototypical target of secretory autophagy, was found to be partially shed within exosomes and microvesicles ${ }^{47,48}$. Other examples include annexin $A 2$, the chaperon $\alpha$-Crystallin $B$, and the high mobility group box 1 protein, which were shown to depend on the functionality of the autophagic machinery as well as the exosome biogenesis pathway for release ${ }^{49-53}$. In most cases, a correlation with the induction of LC3 secretion was demonstrated, highlighting the involvement of a secretory arm of the autophagy pathway in the packaging of cargo molecules in EV. Similarly, we observed a correlation between the extracellular release of LC3 and the enhanced packaging of EMCV in EVs during infection. Moreover, pharmacological induction of autophagic secretion supported the EVmediated release of virions.

Three models have been proposed to explain how secretory autophagy could contribute to the release of EV-enclosed cargo molecules. Firstly, autophagosomes may fuse directly with the plasma membrane to release the autophagosome inner membranous vesicle. Secondly, autophagosomes may fuse with multivesicular bodies (MVBs) or endosomes to result in hybrid compartments, referred to as amphisomes, that are subsequently released ${ }^{29}$. Finally, the autophagic machinery may even be directly recruited to endosomes to aid in EV cargo selection at the MVB limiting membrane, bypassing the need for autophagosome formation altogether ${ }^{54,55}$. In the case of EMCV infection, we observed that the majority of the LC3 released from cells was accessible to degradation by proteinase $\mathrm{K}$. This is compatible with partial disassembly of autophagic membranes in amphisomes, which may liberate LC3 from its membrane confinement and result in the release of LC3 that is exposed to the environment. Conversely, the release of intact autophagosomes as well as the direct recruitment of LC3 to the outer MVB membrane is expected to lead to the selective release of membrane-enclosed LC3. In support of a potential role for amphisomes in the formation of EVenclosed viruses during infection, we found that EVs from EMCV-infected cells were enriched in proteins of both Golgi/ERGIC and endosomal compartments. ERGIC and Golgi have been implicated as important membrane sources for autophagosome formation ${ }^{37-39}$. Structural rearrangements and dispersal of the Golgi and ERGIC were previously reported in EMCV-infected cells, indicating potential recruitment of these membranes to other $\operatorname{sites}^{56}$. We therefore speculate that viral disturbance in the Golgi/ERGIC in presence of the EMCV Leader induces the formation of ERGIC-derived 
autophagosomal membranes that fuse with endosomal compartments, where they become incorporated into budding EV. Endosomal proteins enriched in EVs from EMCV-infected cells included the Lipopolysaccharide-induced tumor necrosis factor-alpha factor (LITAF) and the ESCRTIII complex protein charged multivesicular body protein 2A (CHMP2A). LITAF and CHMP2A have been implicated in both MVB formation and autophagy, and CHMP2A was previously also recognized to play an important role in the EV-mediated release of the picornavirus $\mathrm{HAV}^{57-59}$. Our study provides leads on how virions may be packaged in EVs, but the exact mechanism via which the autophagosomes and endosomal systems intersect and facilitate this process remains to be determined.

Limited knowledge is currently available on the molecular mechanisms that mediate the rerouting of autophagosomes onto a pathway for extracellular release. It has been proposed that specific regulators may be recruited to autophagosomes that enable their preferential fusion with the PM or other non-lysosomal compartments, such as GRASP55 and TRIM16 ${ }^{36,48,60,61}$. For the picornavirus CVB3, we recently identified a role for the transcription factor EB (TFEB), a known regulator of lysosomal exocytosis, in the activation of secretory autophagy ${ }^{62}$. TFEB depletion limited the ability of CVB3 to induce LC3 release during infection, which correlated with an overall decrease in EVenclosed virus release. In contrast, other studies have suggested that autophagic secretion may be a response to defects in autophagosome clearance. Inhibitors of lysosomal function, such as apilimod, were shown to trigger the release of autophagic cargo upon prolonged exposure ${ }^{43,63}$. Examples in pathological settings include the defects in autophagosome clearance that accompany the accumulation of prion-like proteins such as $\alpha$-synuclein, which leads to their export from cells together with LC3 ${ }^{63-68}$. Several viruses are also known to interfere with lysosomal function. In specific, picornaviruses belonging to the enterovirus genus have been reported to disrupt the molecular machinery necessary for autophagosome-lysosome fusion ${ }^{69-71}$. However, our data indicate that the EMCV Leader protein enabled the secretion of autophagosomal cargo without interfering with lysosomal degradation or causing an inherent block in autophagosome-lysosome fusion. Despite an intracellular accumulation of autophagosomes, no lysosomal defects were observed in infected cells. In addition, autolysosome formation in EMCV infected cells could still be enhanced by rapamycin treatment. Hence, the induction of EV-enclosed and autophagic cargo release by EMCV does not appear to be a response to a limitation in cellular degradation capacity caused by the presence of the Leader. Rather, we propose that virus infection leads to specific activation of this secretory pathway. Given the diverse effects of picornavirus species on autophagosome-lysosome fusion, picornaviruses may employ different mechanisms to prevent degradation and enable the secretion of EV-enclosed virions sequestered by the autophagic machinery.

Many viral proteins across the picornaviridae family have evolved strongly overlapping functions despite showing little to no sequence or structural similarities. The class of viral security proteins, to which the EMCV Leader belongs, unify with respect to the host cell processes they target during infection $^{30}$. Follow-up studies should therefore address whether the role of EMCV Leader in the induction of virus-carrying EV that we discovered here also represents a repeating feature among the non-structural viral proteins of this exceptionally large group of viruses. Ultimately, the identification of the viral and host factors involved in the regulation of EV-enclosed release will shed new light on the role of these particles in the viral life cycle and increase our understanding of the intricate relationship between autophagy, EVs, and virus-host interactions. 


\section{Acknowledgements}

We are grateful to dr. Ger Arkesteijn for help with high resolution flow cytometric analysis of EVs, as well as the Center for Cell Imaging Utrecht for allowing access to their facility for confocal imaging and training. We thank dr. Carla Ribeiro for providing antibody against LC3 and dr. Guillaume van Niel for helpful discussions. This work was funded by The Netherlands Organisation for Scientific Research (NWO-ALW grant number ALWOP.351) (https://www.nwo.nl/en) as well as the European Research Council under the European Union's Seventh Framework Programme [FP/2007-2013] / ERC Grant Agreement number [337581] to E.N.M.N.tH. (https://erc.europa.eu/funding/starting-grants). This research was part of the Netherlands X-omics Initiative and partially funded by NWO (Project 184.034.019 to M.A.). The funders had no role in study design, data collection and analysis, decision to publish, or preparation of the manuscript.

\section{Author Contributions}

S.G.vdG., K.A.Y.D., F.J.M.vK. and E.N.M.N.tH. conceived the project. S.G.vdG., K.A.Y.D., F.J.M.vK. and E.N.M.N.tH. wrote the manuscript. S.G.vdG. and K.A.Y.D. designed and performed experiments. S.S.G. and M.A. performed mass spectrometry and M.J.C.vH performed subsequent data analysis. H.H.R. performed confocal imaging of LC3 reporter cells. R.W.W. together with K.A.Y.D. performed and analyzed lysotracker staining. E.N.M.N.tH and F.J.M.vK supervised the project.

\section{Declaration of Interests}

The authors declare no competing interests.

\section{Materials and Methods}

\section{Cells and virus}

Human cervical carcinoma cells (HeLa R19) were a kind gift from $\mathrm{Dr}$ G. Belov (University of Maryland, USA) and baby hamster kidney cells (BHK21, ATTC CCL-10) were obtained from the American Type Culture Collection (Rockville, MD). HeLa R19 cells stably expressing mCherry-EGFP-LC3 were obtained through stable transfection of plasmid DNA (Addgene \#22418), followed by puromycin selection and generation of monoclonal cell populations by limited dilution. Cell lines were cultured in a humidified incubator at $37^{\circ} \mathrm{C}$ and $5 \% \mathrm{CO}_{2}$ in Iscove's modified Dulbecco's medium (IMDM; Lonza, Basel, Switzerland) with $2 \mathrm{mM}$ Ultraglutamine (Lonza), or Dulbecco's Modified Eagle Medium (high glucose, GlutaMAX ${ }^{\mathrm{TM}}$, ThermoFischer Scientific, Waltham, MA, USA), supplemented with $10 \%$ fetal calf serum (FCS; GE Healthcare Bio-Sciences, Chicago, IL), $100 \mathrm{U} / \mathrm{mL}$ penicillin and $100 \mu \mathrm{g} / \mathrm{mL}$ streptomycin (Gibco, Paisley, United Kingdom).

Both EMCV-Wt and EMCV-L ${ }^{\mathrm{Zn}}$ stocks were obtained by transfection of BHK21 cells with in vitro RNA transcripts of the previously described infectious CDNA clone pM16.1, which contains a copy of the EMCV genome with a shortened poly-C tract $^{72}$. The $\mathrm{Zn}$-finger mutation in the Leader was introduced by site-directed mutagenesis as described previously ${ }^{31}$. Virus-containing culture supernatant was harvested after observing virus-induced cytopathogenic effect (CPE) and subjected to three consecutive freeze-thaw cycles. Virus was concentrated from cell-free culture supernatant by highspeed ultracentrifugation through a $30 \%$ sucrose cushion at $80,000 \mathrm{xg}$ for $16 \mathrm{hrs}$ in a SW32 rotor (kfactor 321) (Beckman Coulter, Brea, CA). 


\section{Drug treatments}

To block lysosomal acidification and subsequent autophagosomal degradation, cells were treated with 200 nM bafilomycin A1 (Cayman Chemical, Ann Arbor, MI) from 1 hr p.i. onwards or for the indicated duration of time. To enhance autophagic flux, cells were treated with $300 \mathrm{nM}$ rapamycin (Tocris Bioscience, Bristol, United Kingdom) from $1 \mathrm{hr}$ p.i. onwards. To activate secretory autophagy, cells were pretreated with 500nM apilimod (AChemBlock, Burlingame, CA) for 16 hrs, followed by infection in the presence of apilimod for the indicated duration of time.

\section{Isolation of EVs}

For isolation of EVs from pre-lytic EMCV-infected cells, HeLa R19 cells were seeded in T75 culture flasks and infected the next day at $\mathrm{MOI}=10$. At $1 \mathrm{hr}$ p.i., unbound virus was removed by washing the cells 3 times with PBS, and cells were provided with culture medium containing EV-depleted FCS. To remove EVs from FCS, 30\% FCS in IMDM was ultracentrifuged for 16-20 hrs at 100,000xg in an SW32 rotor (k-factor 256.8 ) and passed through a $0.22 \mu \mathrm{m}$ filter. Cell culture supernatants were collected after indicated culture durations and were sequentially centrifuged at 1-2x 200xg for $10 \mathrm{~min}, 2 \mathrm{x}$ 500xg for $10 \mathrm{~min}$, and 1x 10,000xg for $30 \mathrm{~min}$ to remove cell debris and large EVs. 100K EVs were isolated by ultracentrifugation of $10,000 x g$ supernatant at $100,000 x g$ for 65 min in a SW40 rotor (kfactor 280.3) (Beckman-Coulter). EV-containing 100K ultracentrifugation pellets were resuspended in $20 \mu \mathrm{L}$ PBS containing $0.2 \%$ BSA (cleared from aggregates by ultracentrifugation for 16-20 hrs at $100,000 x g)$. For further purification, resuspended EV pellets were mixed with $60 \%$ iodixanol (Optiprep; Axis-Shield, Oslo, Norway) to a final concentration of $45 \%$ iodixanol and overlaid with a linear gradient of $40 \%-5 \%$ iodixanol in PBS. Density gradients were centrifuged at $192,000 \times$ for $15-$ $18 \mathrm{hrs}$ in a SW40 rotor (k-factor 144.5). Gradient fractions of $1 \mathrm{~mL}$ were collected and densities were assessed by refractometry. Unless otherwise indicated, fractions within the $1.04-1.10 \mathrm{~g} / \mathrm{ml}$ density range were pooled for the characterization of EVs and EV-enclosed virus release based on the buoyant densities validated previously for naked EMCV virions and $\mathrm{EVs}^{5}$.

\section{Confocal microscopy}

HeLa R19 LC3 reporter cells expressing the mCherry-EGFP-LC3 plasmid were infected with EMCV-Wt or EMCV- $\mathrm{L}^{\mathrm{Zn}}$ at $\mathrm{MOI}=10$ for confocal imaging. At the indicated time points, cells were fixed in PBS + 4\% PFA. Cells were imaged using a Leica SPEII/DMI4000 confocal microscope with hardware settings to image GFP and mCherry channels sequentially by 488 and $561 \mathrm{~nm}$ laser excitation using recommended emission spectral filter settings. Acquisition was performed using an ACS APO 63x oil immersion objective (N.A. 1.3) with the pinhole set to 1 airy disk. Data were analyzed using ImageJ software $(1.52 \mathrm{i})^{73}$. For each condition $\geq 7$ images were taken with $2-5$ cells each. Background subtraction was performed using Gaussian blur (sigma 7) and EGFP and mCherry positive puncta were segmented by thresholding. Summed surface area measurements of EGFP and mCherry positive puncta per image field were collected and ratios of these measurements are depicted. For Iysotracker staining, HeLa R19 cells were seeded in FluoroBrite ${ }^{\text {TM }}$ Dulbecco's modified Eagle medium (DMEM; Thermo Fischer Scientific, Waltham, MA, USA), supplemented with 10\% FCS, $1 \%$ P/S and 2 $\mathrm{mM}$ ultraglutamine. Cells were infected at $\mathrm{MOI}=25$ or treated with $200 \mathrm{nM}$ BafA1 and mounted on a Nikon A1R confocal microscope while maintaining cell culture conditions $\left(37^{\circ} \mathrm{C}, 5 \% \mathrm{CO}_{2}\right)$ in a tabletop humidified culture control unit (TOKAI Hit). Live cell imaging of $4 \times 4$ fields of view was performed using a 60x CFI PLAN APO oil objective or a 20x PLAN APO VC air objective with widened pinhole settings (20.43 um) approximately 6 hrs p.i. following the addition of 50 nM LysoTracker Red DND-99 (Thermo Fischer Scientific, Waltham, MA, USA). For quantification of the average lysotracker staining intensity of the cells, images were processed using NIS elements 5.1 general analysis module (Nikon Microsystems). A rolling ball background correction $(6 \mu \mathrm{m})$ and 3 iterations of smoothing were performed before setting a low intensity threshold to segment cells and exclude extracellular areas from analysis (monitored by DIC images and the lysotracker staining). A higher threshold setting was determined using the BafA1 treated controls to exclude segmented areas consisting of cells that had undergone virus-induced lytic cell death (supported by DIC images). 


\section{Flow cytometric analysis of cells}

Cell viability was assessed using 7-AAD viability staining (eBioscience, San Diego, CA) or Fixable Viability Dye efluor ${ }^{\mathrm{TM}} 506$ (eBioscience, San Diego, CA) according to manufacturer's protocols. In short, adherent cells were harvested by trypsinization and pooled with any detached cells recovered from the supernatant following centrifugation at $200 \mathrm{xg}$ for $10 \mathrm{~min}$. For 7-AAD staining cells were washed with phosphate buffered saline (PBS), and stained with $5 \mu \mathrm{L}$ dye per $1 \times 10^{6}$ cells for 5 min at RT. Cells were analyzed using a BD FACS Canto II (BD Biosciences, San Jose, CA) with BD FACS Diva software. For the Fixable Viability Dye eFluor ${ }^{\mathrm{TM}} 506$ labelling, cells were washed twice in ice cold PBS followed by staining on ice for 30 min using a 1:1000 working dilution. Unbound dye was removed by washing with PBS and the cells were fixed in $1 \%$ paraformaldehyde (PFA). Cells were analyzed using a CytoFLEX LX (Beckman Coulter). Data analysis was performed using FlowJo v10.07 software (FlowJo LLC, Ashland, OR). Heat shocked cells were taken along in all experiments as positive control. To this end, cells were incubated for $3 \mathrm{~min}$ at $65^{\circ} \mathrm{C}$ followed by immediate placement on ice for $1 \mathrm{~min}$.

\section{High-resolution flow cytometric analysis of EVs}

For high-resolution flow cytometric analysis of EVs, 100K pelleted EVs were labeled with $30 \mu \mathrm{M}$ CFSE (Invitrogen, Carlsbad, CA) in $50 \mu \mathrm{l}$ PBS for $1 \mathrm{hr}$ at RT. Unbound CFSE was separated from EVs by density gradient centrifugation as described above. EVs in gradient fractions were fixed with $2 \%$ paraformaldehyde for 30 min and diluted in PBS for high-resolution flow cytometric analysis on a BD Influx flow cytometer with optimized configuration, as previously reported ${ }^{32,33}$. In short, thresholding was applied on fluorescence generated by CFSE-labeled EVs passing the $488 \mathrm{~nm}$ laser. Fluorescent $100 \mathrm{~nm}$ and $200 \mathrm{~nm}$ polystyrene beads (FluoSpheres, Invitrogen) were used to calibrate the fluorescence, and forward (FSC) and side (SSC) scattered light settings. Samples were measured at low pressure (sheath fluid: 5 PSI, sample: 4.2 PSI) using a $140 \mu \mathrm{m}$ nozzle with event rates below 10,000 per second. Measurements were acquired in a fixed time window of 30 seconds to allow direct comparison of EV concentrations in parallel samples. Data analysis was performed using FlowJo software or FCS expression v3 (De Novo software, Los Angeles, CA).

\section{End-point dilution}

Intracellular infectivity levels were evaluated in samples of infected HeLa R19 cells that were subjected to 3 consecutive freeze-thaw cycles, followed by centrifugation for $10 \mathrm{~min}$ at $500 \mathrm{xg}$ to remove cell debris. Extracellular infectivity levels in the pre-lytic stage of infection were determined in supernatants of infected cells after a similar pre-clarification step. Infectivity in purified EVs was assessed by direct sampling from EV-containing density gradient fractions. BHK21 cells in 96-well clusters were infected with 3 to 5 -fold serial dilutions of the material described above, and TCID50 values were calculated 3 days after infection using the Spearman-Karber calculation method.

\section{SDS-PAGE and western blotting}

For analysis of cell lysates, cells were lysed in RIPA buffer ( $40 \mathrm{mM}$ Tris-HCl pH 8, 0.5\% sodium deoxycholate, $1 \%$ Triton $\mathrm{X}-100,150 \mathrm{mM}$ sodium chloride, $0.1 \%$ sodium dodecyl sulfate) with a protease inhibitor cocktail (Roche). Lysates were cleared by centrifugation at $16,000 \times g$ for $15 \mathrm{~min}$ and protein concentration was determined using a Pierce BCA assay kit (ThermoScientific, Waltham, $\mathrm{MA}$ ) according to the manufacturer's protocol. Cell lysates or $100 \mathrm{~K}$ ultracentrifugation pellets containing EVs were denatured at $100^{\circ} \mathrm{C}$ for $4 \mathrm{~min}$ in non-reducing Laemmli sample buffer (LSB: 62.5 $\mathrm{mM}$ Tris-HCl pH 6.8, 2\% SDS, $10 \%$ glycerol) for detection of CD63 and flotillin, and reducing LSB (+20 $\mathrm{mM} 2-M e r c a p t o e t h a n o l)$ for detection of LC3 and GAPDH. Proteins were separated on $12.5 \%$ sodium dodecyl sulfate-polyacrylamide gels by electrophoresis (SDS-PAGE) and transferred to Immobilon $0.20 \mu \mathrm{m}$ (LC3, GAPDH) or $0.45 \mu \mathrm{m}$ (CD9, CD63) pore-sized PVDF membranes (Merck Millipore Ltd., Cork, Ireland) by wet transfer. Membranes were incubated with blocking buffer ( $0.2 \%$ fish skin gelatin (FSG; Sigma-Aldrich) (CD9, CD63, LC3, GAPDH) or 4\% BSA (LC3) + 0.1\% Tween-20 in PBS) for 1 $\mathrm{hr}$, and for $>16 \mathrm{hrs}$ at $4^{\circ} \mathrm{C}$ with the following primary antibodies: mouse- $\alpha$-CD63 (1:1000, clone TS63; 
Abcam, Cambridge, United Kingdom), mouse- $\alpha$-Flotillin-1 (1:1000, clone 18/Flotillin-1; BD Biosciences), mouse- $\alpha$-LC3 (1:500, clone 5F10; 1:50, clone 2G6; Nanotools, Teningen, Germany), rabbit- $\alpha$-LC3 (1:1000, polyclonal; MBL international, Woburn, MA), and mouse- $\alpha-G A P D H$ (1:2000, clone mAbcam 9484; Abcam) diluted in blocking buffer. Membranes were subsequently washed with blocking buffer, incubated for $1 \mathrm{hr}$ with HRP-coupled goat-anti-mouse secondary antibody (1:10,000; Jackson ImmunoResearch Labaratories Inc., West Grove, PA) diluted in blocking buffer, and washed again. ECL solution (SuperSignal West Dura Extended Duration Substrate, ThermoScientific) was used for detection on a Bio-Rad ChemiDoc imager and images were analyzed by Image Lab software (BioRad).

\section{Proteinase $\mathrm{K}$ digestion}

$100 \mathrm{~K}$ EV pellets isolated from the supernatants of EMCV-Wt infected cells were resuspended in PBS and incubated in the absence or presence of $0.2 \%$ Triton X-100 for $15 \mathrm{~min}$ at RT. $45 \mathrm{ng} / \mu \mathrm{l}$ Proteinase $\mathrm{K}$ (Roche, Basel, Switzerland) was added followed by a second incubation step at $37^{\circ} \mathrm{C}$ for $30 \mathrm{~min}$. Proteinase $\mathrm{K}$ was inactivated by the addition of $1 \mathrm{mM}$ PMSF (Roche) and samples mixed with LSB for SDS-PAGE and western blot analysis.

\section{Proteomics sample preparation}

For MS analysis, EV-containing density fractions $(1.06-1.13 \mathrm{~g} / \mathrm{ml})$ were diluted in PBS containing $0.1 \%$ BSA and centrifuged at $100,000 \times g$ for $90 \mathrm{~min}$ in an SW32 rotor. EV pellets were resuspended in reducing Laemmli sample buffer and denatured at $100^{\circ} \mathrm{C}$ for $4 \mathrm{~min}$. The protein content of EV preparations was estimated by SDS-PAGE followed by SYPRO ${ }^{\circledR}$ Ruby protein staining densitometry. In short, sample aliquots were used for protein separation on 8-16\% TGX-Criterion gels (Bio-Rad, Hercules, CA). After electrophoresis, gels were fixed in $40 \%$ methanol $/ 10 \%$ acetic acid and stained with SYPRO ${ }^{\circledR}$ Ruby (Invitrogen, Carlsbad, CA), followed by destaining in $10 \%$ methanol/6\% acetic acid. Gels were imaged on a Bio-Rad ChemiDoc imaging system and densitometry quantitation was performed using Image Lab software (Bio-Rad). For LC-MS/MS analysis, EV-protein input was equalized between conditions and proteins were separated by SDS-PAGE on 8-16\% TGX-Criterion gels. Gels were fixed in 50\% methanol/10\% acetic acid, stained with Coomassie Brilliant Blue R-250 (Bio-Rad) and destained sequentially with $40 \%$ methanol/10\% acetic acid and MilliQ. In-gel digestion using trypsin (Promega, Madison, WI) was performed as previously described ${ }^{74}$. After extraction with $100 \%$ acetonitrile the samples were dried and reconstituted in $10 \%$ formic acid/5\% DMSO.

\section{High-resolution LC-MS/MS and data analysis}

The samples were analyzed with a Q-Exactive (Thermo Scientific, Bremen, Germany) connected to an Agilent 1290 Infinity LC system, a trap column of $20 \mathrm{~mm} \times 100 \mu \mathrm{m}$ ID Reprosil C18 (Dr. Maisch, Ammerbuch, Germany) and a $450 \mathrm{~mm} \times 75 \mu \mathrm{m}$ ID Poroshell C18 analytical column (Zorbax, Agilent Technologies, Santa Clara, CA), all packed in-house. Solvent A consisted of $0.1 \mathrm{M}$ acetic acid (Merck, Kenilworth, $\mathrm{NJ}$ ) in Milli-Q (Millipore, Burlington, $\mathrm{MA}$ ), while solvent $\mathrm{B}$ consisted of $0.1 \mathrm{M}$ acetic acid in $80 \%$ acetonitrile (Biosolve, Valkenswaard, The Netherlands). Trapping was performed at a flow rate of $5 \mathrm{\mu l} / \mathrm{min}$ for $10 \mathrm{~min}$ and peptides were eluted at $100 \mathrm{nl} / \mathrm{min}$ for $55 \mathrm{~min}$. The mass spectrometer was operated in data-dependent mode to automatically switch between MS and MS/MS. For the MS/MS analysis the fifteen most intense ions in the survey scan ( 375 to $1600 \mathrm{~m} / \mathrm{z}$, resolution 60,000, AGC target 3e6) were subjected to HCD fragmentation (resolution 30,000, AGC target 1e5), with the normalized collision energy set to $27 \%$ for $\mathrm{HCD}$. The signal threshold for triggering an MS/MS event was set to 500 counts. The low mass cut-off for HCD was set to $180 \mathrm{~m} / \mathrm{z}$. Charge state screening was enabled, and precursors with unknown charge state or a charge state of 1, $6-8$ and $>8$ were excluded. Dynamic exclusion was enabled (exclusion size list 500, exclusion duration 8 s). MS data 
processing and analysis MS raw data were processed with Proteome Discoverer (version 1.4.1.14, Thermo Fisher). Peptide identification was performed using Mascot 2.3 (Matrix Science, UK). Peak lists were generated from the raw data files using Proteome Discoverer version 1.4.1.14 (Thermo Scientific, Bremen) and searched against the current (Consulted in July 2018) UniProtKB/Swiss-Prot database (all entries for identification of EMCV proteins, Homo sapiens for identification of host proteins), supplemented with frequently observed contaminants. Trypsin was chosen as enzyme and two missed cleavages were allowed. Carbamidomethylation (C) was set as a fixed modification and oxidation (M) was set as variable modification. The searches were performed using a peptide mass tolerance of $50 \mathrm{ppm}$ and a product ion tolerance of $0.05 \mathrm{Da}$, followed by data filtering using percolator, resulting in $1 \%$ false discovery rate (FDR). Only ranked 1 PSMs with Mascot scores $>20$ were accepted. Functional enrichment analysis was performed for cellular component and biological process using PANTHER (via http://geneontology.org). By hierarchical sorting of analysis results, only the most specific subclasses of GO-terms are presented, while redundant parental terms are excluded. Enriched GO-terms were ranked by fold enrichment versus the genome. Only significantly enriched GO terms are presented. P-values are determined by Fisher's exact test with Bonferroni correction for multiple testing. Protein-protein interactions were analyzed using the STRING database version 11.0 (https://string-db.org/), with the minimum required interaction score set to medium confidence $(0.400)$. 


\section{References}

1. Feng, Z. et al. A pathogenic picornavirus acquires an envelope by hijacking cellular membranes. Nature 496, 367-71 (2013).

2. Robinson, S. M. et al. Coxsackievirus B exits the host cell in shed microvesicles displaying autophagosomal markers. PLoS pathogens 10, e1004045 (2014).

3. Chen, Y.-H. et al. Phosphatidylserine vesicles enable efficient en bloc transmission of enteroviruses. Cell 160, 619-30 (2015).

4. Gu, J. et al. Exosomes cloak the virion to transmit Enterovirus 71 non-lytically. Virulence 11, 32-38 (2020).

5. van der Grein, S. G. et al. Picornavirus infection induces temporal release of multiple extracellular vesicle subsets that differ in molecular composition and infectious potential. PLoS Pathogens 15, (2019).

6. Colombo, M., Raposo, G. \& Théry, C. Biogenesis, secretion, and intercellular interactions of exosomes and other extracellular vesicles. Annual review of cell and developmental biology 30, 255-89 (2014).

7. Yáñez-Mó, M. et al. Biological properties of extracellular vesicles and their physiological functions. Journal of Extracellular Vesicles 4, 27066 (2015).

8. Takahashi, M. et al. Monoclonal antibodies raised against the ORF3 protein of hepatitis e virus (HEV) can capture HEV particles in culture supernatant and serum but not those in feces. Archives of Virology 153, 1703-1713 (2008).

9. Takahashi, M. et al. Hepatitis E Virus (HEV) strains in serum samples can replicate efficiently in cultured cells despite the coexistence of HEV antibodies: characterization of HEV virions in blood circulation. Journal of clinical microbiology 48, 1112-25 (2010).

10. Santiana, M. et al. Vesicle-Cloaked Virus Clusters Are Optimal Units for Inter-organismal Viral Transmission. Cell Host and Microbe 24, 208-220.e8 (2018).

11. Mao, L. et al. Enterovirus 71 transmission by exosomes establishes a productive infection in human neuroblastoma cells. Virus genes 52, 189-94 (2016).

12. Nagashima, S. et al. Characterization of the Quasi-Enveloped Hepatitis E Virus Particles Released by the Cellular Exosomal Pathway. Journal of Virology 91, (2017).

13. Chapuy-Regaud, S. et al. Characterization of the lipid envelope of exosome encapsulated HEV particles protected from the immune response. Biochimie 141, 70-79 (2017).

14. Hudry, E. et al. Exosome-associated AAV vector as a robust and convenient neuroscience tool. Gene therapy 23, 380-92 (2016).

15. Gu, J. et al. A mouse model for infection with enterovirus A71 in small extracellular vesicles. mSphere 5, (2020).

16. Das, A. et al. TIM1 (HAVCR1) Is Not Essential for Cellular Entry of Either Quasi-enveloped or Naked Hepatitis A Virions. mBio 8, e00969-17 (2017). 
17. Fu, Y. et al. Exosome-mediated miR-146a transfer suppresses type I interferon response and facilitates EV71 infection. PLoS pathogens 13, e1006611 (2017).

18. Yin, X., Ambardekar, C., Lu, Y. \& Feng, Z. Distinct Entry Mechanisms for Nonenveloped and Quasi-Enveloped Hepatitis E Viruses. Journal of virology 90, 4232-42 (2016).

19. O'Hara, B. A., Morris-Love, J., Gee, G. v., Haley, S. A. \& Atwood, W. J. JC Virus infected choroid plexus epithelial cells produce extracellular vesicles that infect glial cells independently of the virus attachment receptor. PLoS Pathogens 16, (2020).

20. Altan-Bonnet, N. \& Chen, Y.-H. Intercellular Transmission of Viral Populations with Vesicles. Journal of Virology 89, 12242-12244 (2015).

21. Feng, Z. et al. Human pDCs preferentially sense enveloped hepatitis A virions. The Journal of clinical investigation 125, 169-76 (2015).

22. Jeon, H. et al. Extracellular vesicles from KSHV-infected cells stimulate antiviral immune response through mitochondrial DNA. Frontiers in Immunology 10, (2019).

23. Deschamps, T. \& Kalamvoki, M. Extracellular Vesicles Released by Herpes Simplex Virus 1Infected Cells Block Virus Replication in Recipient Cells in a STING-Dependent Manner. Journal of Virology 92, (2018).

24. Sin, J., Mclntyre, L., Stotland, A., Feuer, R. \& Gottlieb, R. A. Coxsackievirus B Escapes the Infected Cell in Ejected Mitophagosomes. Journal of virology 91, e01347-17 (2017).

25. Too, I. H. K. et al. Enterovirus 71 infection of motor neuron-like NSC-34 cells undergoes a nonlytic exit pathway. Scientific reports 6, 36983 (2016).

26. Parzych, K. R. \& Klionsky, D. J. An overview of autophagy: Morphology, mechanism, and regulation. Antioxidants and Redox Signaling vol. 20 460-473 (2014).

27. Jackson, W. T. et al. Subversion of cellular autophagosomal machinery by RNA viruses. PLoS biology 3, e156 (2005).

28. Zhang, Y., Li, Z., Ge, X., Guo, X. \& Yang, H. Autophagy promotes the replication of encephalomyocarditis virus in host cells. Autophagy 7, 613-628 (2011).

29. Ponpuak, M. et al. Secretory autophagy. Current opinion in cell biology 35, 106-16 (2015).

30. Agol, V. I. \& Gmyl, A. P. Viral security proteins: Counteracting host defences. Nature Reviews Microbiology vol. 8 867-878 (2010).

31. Hato, S. v. et al. The mengovirus leader protein blocks interferon- $\alpha / \beta$ gene transcription and inhibits activation of interferon regulatory factor 3. Cellular Microbiology 9, 2921-2930 (2007).

32. Nolte-'t Hoen, E. N. M. et al. Quantitative and qualitative flow cytometric analysis of nanosized cell-derived membrane vesicles. Nanomedicine : nanotechnology, biology, and medicine 8, 712-20 (2012).

33. van der Vlist, E. J., Nolte-'t Hoen, E. N. M., Stoorvogel, W., Arkesteijn, G. J. A. \& Wauben, M. $\mathrm{H}$. M. Fluorescent labeling of nano-sized vesicles released by cells and subsequent quantitative and qualitative analysis by high-resolution flow cytometry. Nature protocols 7 , 1311-26 (2012). 
34. Wei, Y. et al. Pyruvate kinase type M2 promotes tumour cell exosome release via phosphorylating synaptosome-associated protein 23. Nature Communications 8, (2017).

35. Verweij, F. J. et al. Quantifying exosome secretion from single cells reveals a modulatory role for GPCR signaling. Journal of Cell Biology 217, 1129-1142 (2018).

36. Kimura, T. et al. Dedicated SNARE $s$ and specialized TRIM cargo receptors mediate secretory autophagy . The EMBO Journal 36, 42-60 (2017).

37. Geng, J. \& Klionsky, D. J. The Golgi as a potential membrane source for autophagy. Autophagy vol. 6 950-951 (2010).

38. Ge, L., Melville, D., Zhang, M. \& Schekman, R. The ER-Golgi intermediate compartment is a key membrane source for the LC3 lipidation step of autophagosome biogenesis. eLife 2013, (2013).

39. Ge, L. \& Schekman, R. The ER-Golgi intermediate compartment feeds the phagophore membrane. Autophagy 10, 170-172 (2014).

40. Agrotis, A., Pengo, N., Burden, J. J. \& Ketteler, R. Redundancy of human ATG4 protease isoforms in autophagy and LC3/GABARAP processing revealed in cells. Autophagy 15, 976-997 (2019).

41. Wang, W., Chen, Z., Billiar, T. R., Stang, M. T. \& Gao, W. The Carboxyl-Terminal Amino Acids Render Pro-Human LC3B Migration Similar to Lipidated LC3B in SDS-PAGE. PLOS ONE 8, (2013).

42. Alirezaei, M., Flynn, C. T., Wood, M. R., Harkins, S. \& Whitton, J. L. Coxsackievirus can exploit LC3 in both autophagy-dependent and -independent manners in vivo. Autophagy 11, 13891407 (2015).

43. Hessvik, N. P. et al. PIKfyve inhibition increases exosome release and induces secretory autophagy. Cellular and Molecular Life Sciences 73, 4717-4737 (2016).

44. Romanova, L. I. et al. Antiapoptotic Activity of the Cardiovirus Leader Protein, a Viral "Security" Protein. Journal of Virology 83, 7273-7284 (2009).

45. Borghese, F. \& Michiels, T. The Leader Protein of Cardioviruses Inhibits Stress Granule Assembly. Journal of Virology 85, 9614-9622 (2011).

46. Mao, L. et al. Enterovirus 71 transmission by exosomes establishes a productive infection in human neuroblastoma cells. Virus Genes 52, 189-194 (2016).

47. Lopez-Castejon, G. \& Brough, D. Understanding the mechanism of IL-1 $\beta$ secretion. Cytokine and Growth Factor Reviews vol. 22 189-195 (2011).

48. Dupont, N. et al. Autophagy-based unconventional secretory pathway for extracellular delivery of IL-1ß. The EMBO journal 30, 4701-11 (2011).

49. Sreekumar, P. G. et al. $\alpha$ B crystallin is apically secreted within exosomes by polarized human retinal pigment epithelium and provides neuroprotection to adjacent cells. PLOS ONE 5, 12578 (2010).

50. Kim, Y. H. et al. Secretory autophagy machinery and vesicular trafficking are involved in HMGB1 secretion. Autophagy 1-18 (2020) doi:10.1080/15548627.2020.1826690. 
51. Fang, Y. T., Lin, C. F., Wang, C. Y., Anderson, R. \& Lin, Y. S. Interferon- $\gamma$ stimulates p11dependent surface expression of annexin $A 2$ in lung epithelial cells to enhance phagocytosis. Journal of Cellular Physiology 227, 2775-2787 (2012).

52. Chen, $\mathrm{Y}$. da et al. Exophagy of annexin A2 via RAB11, RAB8A and RAB27A in IFN- $\gamma$-stimulated lung epithelial cells. Scientific Reports 7, (2017).

53. D'Agostino, M. et al. Unconventional secretion of $\alpha$-Crystallin B requires the Autophagic pathway and is controlled by phosphorylation of its serine 59 residue. Scientific Reports 9 , (2019).

54. Guo, H. et al. Atg5 Disassociates the V1V0-ATPase to Promote Exosome Production and Tumor Metastasis Independent of Canonical Macroautophagy. Developmental Cell 43, 716730.e7 (2017).

55. Leidal, A. M. et al. The LC3-conjugation machinery specifies the loading of RNA-binding proteins into extracellular vesicles. Nature Cell Biology 22, 187-199 (2020).

56. Dorobantu, C. M. et al. Modulation of the Host Lipid Landscape to Promote RNA Virus Replication: The Picornavirus Encephalomyocarditis Virus Converges on the Pathway Used by Hepatitis C Virus. PLoS Pathogens 11, (2015).

57. Takahashi, Y. et al. An autophagy assay reveals the ESCRT-III component CHMP2A as a regulator of phagophore closure. Nature Communications 9, (2018).

58. McKnight, K. L. et al. Protein composition of the hepatitis A virus quasi-envelope. Proceedings of the National Academy of Sciences of the United States of America 114, 6587-6592 (2017).

59. Bertolo, C. et al. LITAF, a BCL6 target gene, regulates autophagy in mature B-cell lymphomas. British Journal of Haematology 162, 621-630 (2013).

60. Gee, H. Y., Noh, S. H., Tang, B. L., Kim, K. H. \& Lee, M. G. Rescue of $\Delta f 508$-CFTR trafficking via a GRASP-dependent unconventional secretion pathway. Cell 146, 746-760 (2011).

61. Nüchel, J. et al. TGFB1 is secreted through an unconventional pathway dependent on the autophagic machinery and cytoskeletal regulators. Autophagy 14, 465-486 (2018).

62. Giansanti, P. et al. Dynamic remodelling of the human host cell proteome and phosphoproteome upon enterovirus infection. Nature Communications 11, (2020).

63. Minakaki, G. et al. Autophagy inhibition promotes SNCA/alpha-synuclein release and transfer via extracellular vesicles with a hybrid autophagosome-exosome-like phenotype. Autophagy 1-22 (2018) doi:10.1080/15548627.2017.1395992.

64. Danzer, K. M. et al. Exosomal cell-to-cell transmission of alpha synuclein oligomers. Molecular neurodegeneration 7, 42 (2012).

65. Alvarez-Erviti, L. et al. Lysosomal dysfunction increases exosome-mediated alpha-synuclein release and transmission. Neurobiology of Disease 42, 360-367 (2011).

66. Koh, J. Y., Kim, H. N., Hwang, J. J., Kim, Y. H. \& Park, S. E. Lysosomal dysfunction in proteinopathic neurodegenerative disorders: Possible therapeutic roles of cAMP and zinc. Molecular Brain vol. 12 1-11 (2019). 
67. Sarkar, S., Olsen, A. L., Sygnecka, K., Lohr, K. M. \& Feany, M. B. $\alpha$-synuclein impairs autophagosome maturation through abnormal actin stabilization. PLOS Genetics 17, e1009359 (2021).

68. Yang, Y., Qin, M., Bao, P., Xu, W. \& Xu, J. Secretory carrier membrane protein 5 is an autophagy inhibitor that promotes the secretion of $\alpha$-synuclein via exosome. PLOS ONE 12, (2017).

69. Mohamud, Y. et al. Enteroviral Infection Inhibits Autophagic Flux via Disruption of the SNARE Complex to Enhance Viral Replication. Cell Reports 22, 3292-3303 (2018).

70. Tian, L. et al. The cytotoxicity of coxsackievirus B3 is associated with a blockage of autophagic flux mediated by reduced syntaxin 17 expression article. Cell Death and Disease 9, (2018).

71. Shi, Y. et al. Coxsackievirus A16 elicits incomplete autophagy involving the mTOR and ERK pathways. PLOS ONE 10, (2015).

72. Duke, G. M. \& Palmenberg, A. C. Cloning and synthesis of infectious cardiovirus RNAs containing short, discrete poly(C) tracts. Journal of Virology 63, 1822-1826 (1989).

73. Schindelin, J. et al. Fiji: An open-source platform for biological-image analysis. Nature Methods vol. 9 676-682 (2012).

74. Shevchenko, A., Wilm, M., Vorm, O. \& Mann, M. Mass spectrometric sequencing of proteins from silver-stained polyacrylamide gels. Analytical Chemistry 68, 850-858 (1996). 


\section{Figure legends}

Figure 1. Inactivation of the Leader protein reduces EV-enclosed virus release by EMCV-infected cells. (A-B) EMCV-Wt and EMCV-L ${ }^{2 n}$-infected HeLa cells and corresponding cell culture supernatants were harvested at the indicated timepoints after infection. Line graphs display the increase in intracellular virus titers $(A)$ and virus titers in the cell culture supernatant (B) over time, as determined by end-point dilution. Depicted are mean values $\pm S D$ from $n=3$ independent experiments. (C) EVs were isolated from EMCV-Wt and EMCV- $\mathrm{L}^{\mathrm{Zn}}$ infected cell culture supernatants at $8 \mathrm{hrs}$ p.i. by differential (ultra)centrifugation. The resulting 100,000xg pelleted EVs were separated from naked virions using density gradient centrifugation and the amount of EV-associated infectivity was determined by end-point dilution assay. Bars display the mean fold infectivity of EMCV-L ${ }^{\mathrm{Zn}} \mathrm{EVS}$ relative to $E M C V-W t E V s \pm S D$ from $n=5$ independent experiments. $* * * p<0.0005$, as assessed by a one sample t-test. (D) EVs released by cells infected with either EMCV-Wt or EMCV-L ${ }^{2 n}$ were isolated and EV protein composition was analyzed by LC-MS/MS. The number of unique EMCV peptides identified within and outside of the capsid-coding region are indicated.

Figure 2. The EMCV Leader is required for the induction of an infectious EV subpopulation with FSC-high phenotype. (A-E) EVs were isolated from cell culture supernatants of EMCV-Wt and EMCV$\mathrm{L}^{\mathrm{Zn}}$ infected cells by differential centrifugation and density gradient centrifugation at indicated timepoints p.i., or from mock-infected cells. EVs were fluorescently labeled with CFSE and analyzed by high-resolution flow cytometry. (A-B) Depicted is the increase over time of EV numbers present in $1.06-1.10 \mathrm{~g} / \mathrm{ml}$ fractions measured in a fixed time window of 30 seconds $(A)$ and the number of EVs present in those density fractions at $8 \mathrm{hrs}$ p.i. (B). (C) Representative FSC/SSC dot plots are depicted of the samples in (B). Gates delineate FSC-high and FSC-low EVs. (D-E) Presented are the percentage of FSC-high EVs of the total EVs present in the $1.08 \mathrm{~g} / \mathrm{ml}$ density fraction at indicated timepoints (D) and the percentage of FSC-high EVs in the $1.08 \mathrm{~g} / \mathrm{ml}$ fraction at $8 \mathrm{hrs} \mathrm{p.i.} \mathrm{(E).} \mathrm{For} \mathrm{A} \mathrm{and} \mathrm{D} \mathrm{a}$ representative image of $n=2$ individual experiments is shown. For $B$ and $E$ mean values $\pm S D$ are presented for $\mathrm{n}=4$ independent experiments. ${ }^{*} \mathrm{p}<0.05,{ }^{* *} \mathrm{p}<0.005$, as assessed by one-way ANOVA with Tukey's multiple comparisons test.

Figure 3. Analysis of the EV proteome supports a Leader-dependent role for secretory autophagy in the formation of virus-induced EV. EVs released by cells infected with either EMCV-Wt or EMCV-L ${ }^{\mathrm{Zn}}$ were isolated and EV protein composition was analyzed by LC-MS/MS. (A) Venn diagram depicting the number of unique and overlapping host proteins identified in at least 2 out of 3 replicate preparations of EMCV-Wt and EMCV-L ${ }^{2 n} E V s$. (B-E) Functional enrichment analysis for the 44 unique proteins in EMCV-Wt EVs was combined with evaluation of protein-protein interactions using the STRING database. (B,D) Functional annotations are listed for the $\mathrm{GO}$ domains cellular component (B) and biological process (D). Bars depict the fold enrichment for the indicated GO-terms compared to the genome, with concomitant $p$-values as assessed by Fisher's exact test with Bonferroni correction for multiple testing. $(C, E)$ Proteins in interactome networks are indicated by their gene name and thickness of the connecting lines correlates with the strength of data support for an interaction. Color-coding of individual proteins corresponds to their association with $\mathrm{GO}$ terms that are enriched $>10$-fold compared to the genome for cellular component (C) or biological process (E). (F) 100K EV isolates from equal numbers of EV-producing cells were analyzed by western blotting for the presence of LC3 and CD63. (G) 100K EV pellets from EMCV-Wt infected cells were treated with proteinase $\mathrm{K}$ in the absence or presence of $0.2 \%$ triton and probed for the presence of LC3 as well as the intraluminal EV marker flotillin. Representative images of $n=2$ independent experiments are shown.

Figure 4. The EMCV Leader promotes the accumulation of autophagosomes during infection. (A) Mock infected cells and cells infected with EMCV-Wt or EMCV-L ${ }^{2 n}$ were harvested $8 \mathrm{hrs}$ p.i. Whole cell lysates were analyzed for conversion of LC3-I to LC3-II by western blotting. For interpretation of LC3 
banding patterns, positive controls (PC) for LC3-I and LC3-II were included, and the LC3-II:LC3-I ratios calculated. GAPDH was included as loading control. *Indicates a detected LC3 band with intermediate size. (B) Schematic overview of the mCherry-EGFP-LC3 autophagy reporter system. Using spot detection on LC3 reporter cells imaged by confocal microscopy, the accumulation of LC3 in autophagosomal vs. autolysosomal compartments can be monitored. Whereas autophagosomes appear as mCherry+EGFP+LC3+ puncta, autolysosomes are detected as mCherry+EGFP-LC3+ puncta due to a loss of EGFP fluorescence in the acidic environment of the autolysosome lumen. (C) LC3reporter cells were infected with EMCV-Wt or EMCV- $\mathrm{L}^{\mathrm{Zn}}$ for the indicated time or treated with bafA1 for $6 \mathrm{hrs}$ and imaged for EGFP and mCherry fluorescence using confocal microscopy. Bar graphs display a quantification of the ratio between the total surface area of EGFP and mCherry positive puncta as a measure for the ratio between the autophagosomes and autolysosomes present in the cells. Indicated are mean values \pm SEM of $\geq 7$ separate images with 2-5 cells each from a

representative of $\mathrm{n}=3$ individual experiments. ${ }^{* *} \mathrm{p}<0.005,{ }^{* * *} \mathrm{p}<0.0005, * * * * \mathrm{p}<0.00005$ as assessed by one-way ANOVA with Dunnett's multiple comparison test. Scale bar $=20 \mu \mathrm{m}$. (D) Infected cells were stained with $50 \mathrm{nM}$ lysotracker and compared to mock infected cells or bafA1 treated controls $6 \mathrm{hrs} \mathrm{p.i.} \mathrm{using} \mathrm{live} \mathrm{cell} \mathrm{confocal} \mathrm{imaging.} \mathrm{Depicted} \mathrm{are} \mathrm{representative} \mathrm{images} \mathrm{showing} \mathrm{lysotracker}$ fluorescence staining. Bar graphs display the average cellular staining intensity relative to uninfected controls. Indicated are mean values \pm SD of 5 independent experiments. Scale bar $=30 \mu \mathrm{m}$. ${ }^{*} p<0.05$, as assessed by an unpaired t-test.

Figure 5. Enhancing autophagic degradation does not inhibit Leader-induced secretory autophagy and EV-enclosed virus release. (A) EMCV-Wt infected cells were treated with $200 \mathrm{nM}$ rapamycin $1 \mathrm{hr}$ p.i. onwards to increase autophagic flux and enhance autolysosome formation. (A) Representative confocal microscopy images of infected mCherry-EGFP-LC3 reporter cells 6 hrs p.i. Bar graphs display the ratio between the total surface area of EGFP and mCherry positive puncta, that correspond to autophagosomes (EGFP+) and autophagosomes/autolysosomes (mCherry+). Indicated are mean values \pm SEM of $\geq 7$ separate images with $2-5$ cells each from a representative of $n=3$ individual experiments. ${ }^{* *} p<0.005,{ }^{* * *} p<0.0005$, as assessed by one-way ANOVA with Dunnett's multiple comparison test. Scale bar $=10 \mu \mathrm{m}$. (B) Western blot analysis of LC3 release detected in 100K EV pellets $8 \mathrm{hrs}$ p.i. (C-D) EVs were isolated from cell culture supernatants by sequential ultracentrifugation steps at 10,000xg (10K) and 100,000 xg (100K) centrifugal force, and purified using density gradient centrifugation. Depicted is the total amount of EV-associated infectivity (C) and its distribution over $100 \mathrm{~K}$ vs. $10 \mathrm{~K}$ EVs (D) as determined by end-point dilution assay. $n s, p>0.05$ as assessed by an unpaired t-test.

Figure 6. Apilimod reactivates secretory autophagy and promotes EV-enclosed virus release in the absence of the EMCV Leader. (A) EMCV- $\mathrm{L}^{\mathrm{Zn}}$ infected cells were treated with $500 \mathrm{nM}$ apilimod for 16 hrs prior to infection or $200 \mathrm{nM}$ BafA1 $1 \mathrm{hr}$ p.i. onwards. Cell culture supernatants were harvested 8 hrs p.i. and subjected to differential ultracentrifugation. The resulting $100,000 \times \mathrm{xg}$ pellets were analyzed by western blot for the presence of extracellular released LC3. Depicted is a representative image of $n=2$ independent experiments. (B) $100 \mathrm{~K}$ pelleted EVs were labelled with CFSE, purified by density gradient centrifugation, and analyzed by high resolution flow cytometry. Bars indicate the mean fold increase in $\mathrm{EV}$ release relative to untreated controls $\pm S D$ from $n=3-4$ independent experiments. (C-D) Mean fold increase in EV-enclosed infectivity (C) and intracellular virus titers (D) relative to untreated controls \pm SD corresponding to the samples depicted in (B) as determined by end-point dilution assay. ${ }^{*} p<0.05$, as assessed by a one-sample t-test. 


\section{Supplementary figure legends}

Supplementary figure 1. Non-lytic virus release by EMCV-Wt vs. EMCV- $\mathrm{L}^{\mathrm{Zn}}$ infected cells. (A) Extracellular virus release by EMCV-Wt and EMCV- $\mathrm{L}^{\mathrm{Zn}}$ infected cells depicted as percentage of the total virus (combined intracellular and extracellular titers) produced at the indicated timepoints. The mean percentages $\pm S D$ of $n=9$ experiments for EMCV-Wt and EMCV- $\mathrm{L}^{\mathrm{Zn}}$ at 8 hrs p.i. and $\mathrm{n}=5$ experiments for EMCV-L $\mathrm{L}^{\mathrm{Zn}}$ at $12 \mathrm{hrs}$ p.i. are shown. ${ }^{* *} \mathrm{p}<0.005,{ }^{* * *} \mathrm{p}<0.0005$, as assessed by oneway ANOVA with Dunnett's multiple comparisons test. (B) EV-enclosed virus release was determined 8 hours p.i. by end-point dilution of density gradient purified EV isolated at 100,000xg. Depicted is the fold difference in $\mathrm{EV}$-enclosed virus release induced by $\mathrm{EMCV}-\mathrm{L}^{\mathrm{Zn}}$ relative to $\mathrm{EMCV}-\mathrm{Wt}$ after normalization for intracellular virus titers $\pm S D(n=5) .{ }^{* * *} p<0.0005$, as assessed by a one-sample $t-$ test.

Supplemental figure 2. The impact of rapamycin treatment on EMCV-Wt infected cells. (A) Cells were infected with EMCV-Wt for $8 \mathrm{hrs}$ and treated or not with rapamycin from $1 \mathrm{hr}$ p.i. onwards, after which intracellular virus production was assessed by end-point dilution. Indicated are mean values $\pm S D$ of $n=5$ individual experiments. (B) Viability of cells treated as in (A) was determined by flow cytometric analysis of fluorescently stained dead cells. The percentage of viable cells is depicted for $n=2$ independent experiments.

Supplementary figure 3. The impact of apilimod and bafilomycin treatment on the viability of EMCV- $\mathrm{L}^{\mathrm{Zn}}$ infected cells. (A) EMCV- $\mathrm{L}^{\mathrm{Zn}}$ infected cells were treated with $500 \mathrm{nM}$ apilimod for $16 \mathrm{hrs}$ prior to infection or $200 \mathrm{nM}$ bafA1 $1 \mathrm{hr}$ p.i. onwards. Cell viability, as assessed by live-dead staining and flow cytometric analysis, was compared to untreated EMCV-L ${ }^{\mathrm{Zn}}$ infected cells or uninfected controls. Indicated are the mean percentage of viable cells $\pm S D$ of $n=2-5$ individual experiments.

\section{Supplementary table legend}

Supplementary table 1. Proteins identified in EVs by LC-MS/MS. Proteins detected in density gradient purified $100 \mathrm{~K}$ EV preparations from EMCV-Wt, EMCV- ${ }^{2 \mathrm{n}}$ infected, or mock-infected cells are listed based on their exclusive or shared identification among the different sample conditions. For protein identification, the MS-generated data was searched against the UniProtKB/Swiss-Prot database (all entries). Proteins listed were identified in at least $2 / 3$ independent replicates. 
bioRxiv preprint doa: https://doi.org/10.1101/2021.05.04.442556; this version posted May 4, 2021. The copyright holder for this preprint (which was not certified by peer review) is the author/funder. All rights reserved. No reuse allowed without permission.

Figure 1

a

Pre-lytic intracellular virus

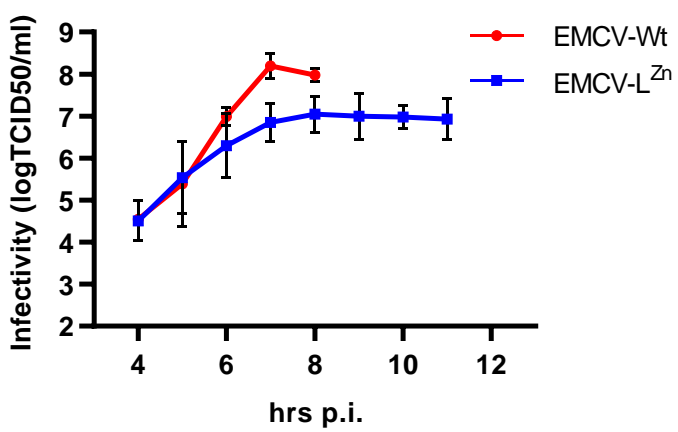

C

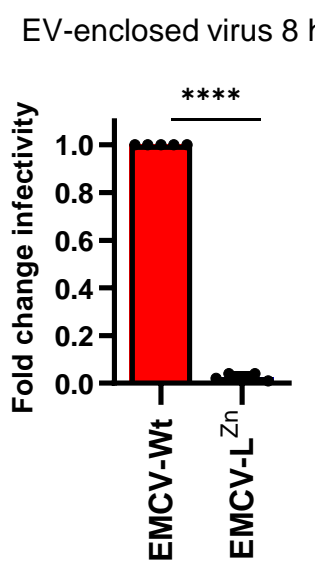

b

Pre-lytic extracellular virus

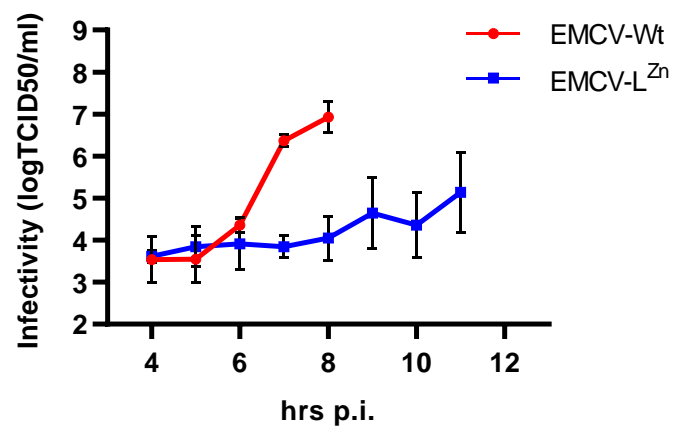

d

Virus peptides in IVs

\begin{tabular}{lll}
\hline & $\begin{array}{l}\text { EMCV-Wt } \\
\text { ENs }\end{array}$ & $\begin{array}{l}\text { EMCV-LZn } \\
\text { ENs }\end{array}$ \\
\hline $\begin{array}{l}\text { Within capsid- } \\
\text { coding region }\end{array}$ & 13 & 0 \\
$\begin{array}{l}\text { Outside capsid- } \\
\text { coding region }\end{array}$ & 4 & 0 \\
\hline
\end{tabular}




\section{Figure 2}

a

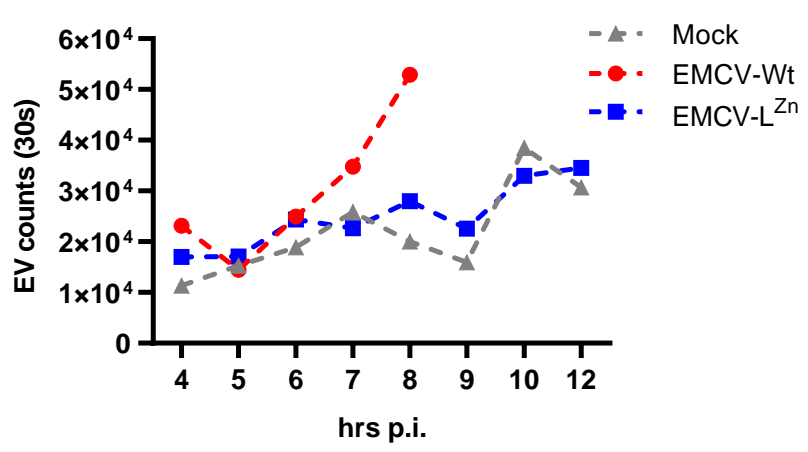

C

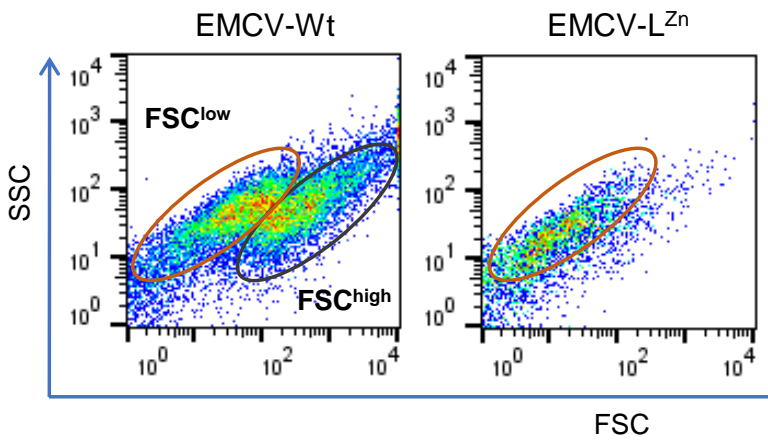

d

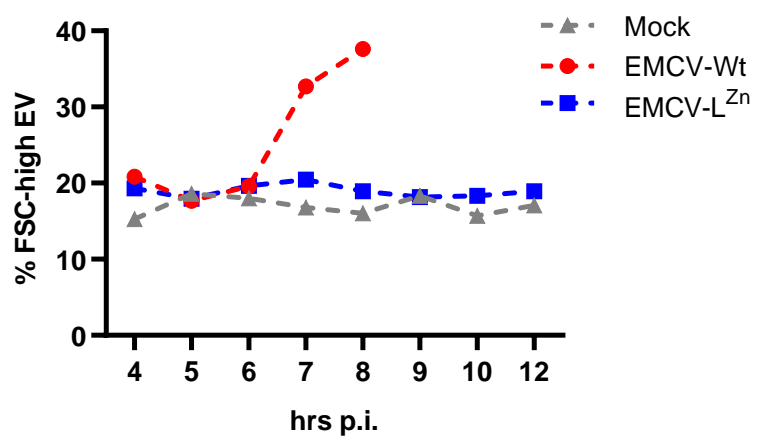

b $\quad 8 \mathrm{hrs}$ p.i.

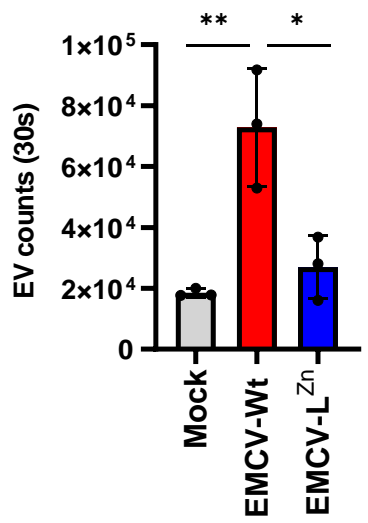

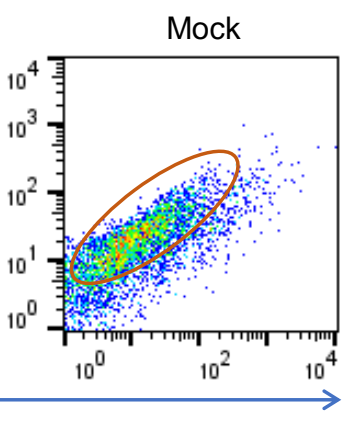

e

8 hrs p.i.

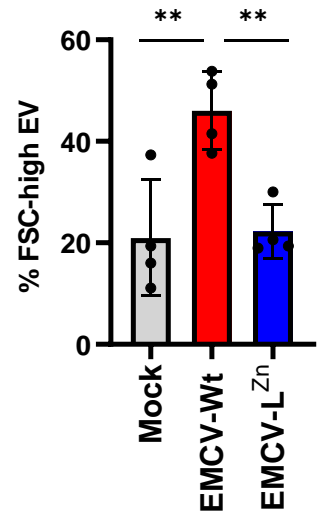




\section{Figure 3}

a

Host proteins in EVs

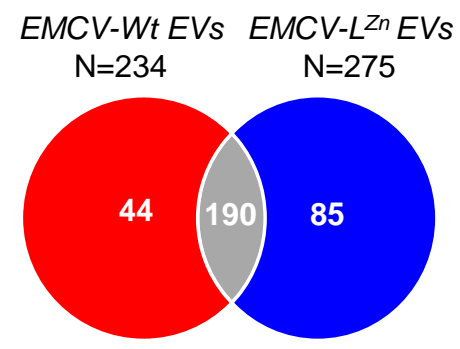

b Cellular component (EMCV-Wt EVs $n=44)$

d Biological process (EMCV-Wt EVs $n=44)$
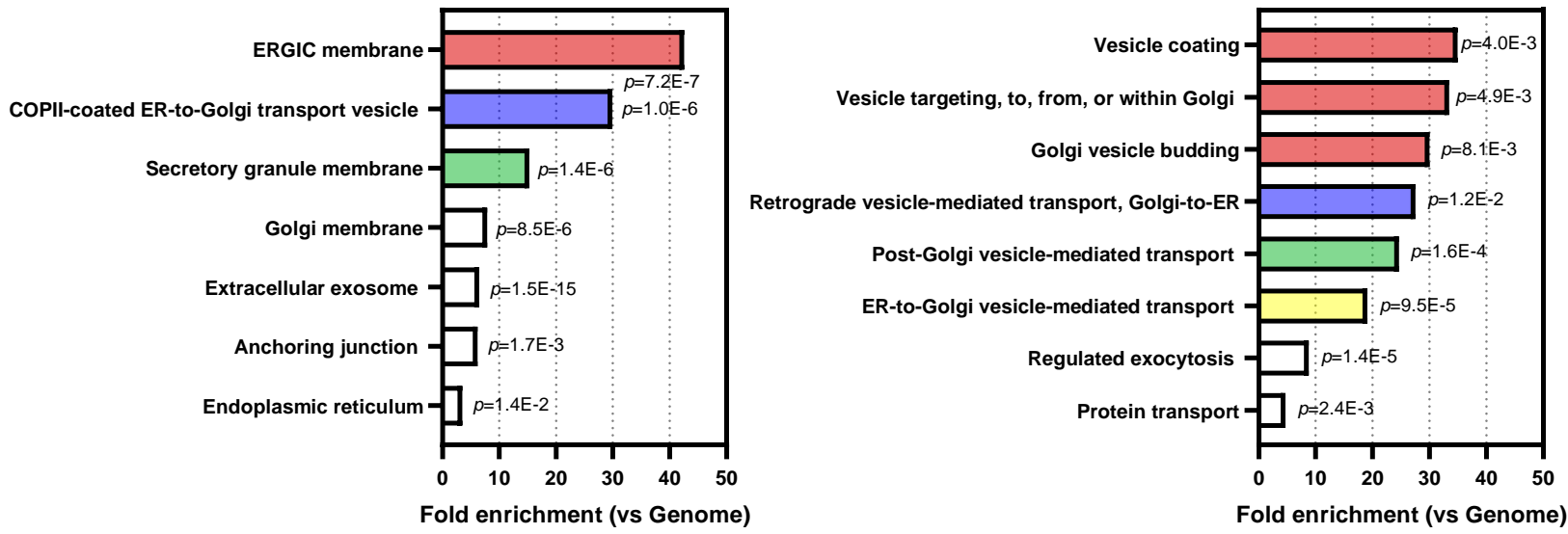

C

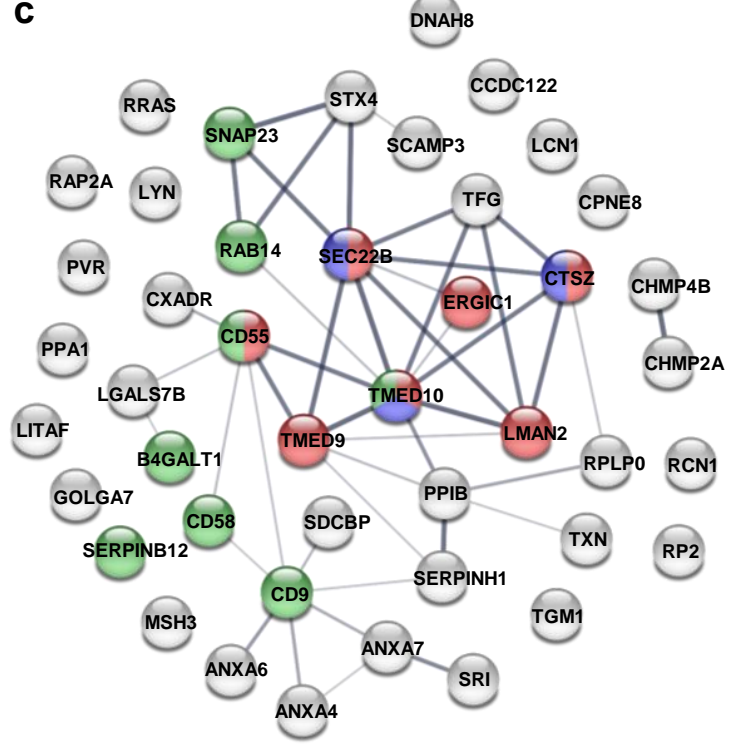

f

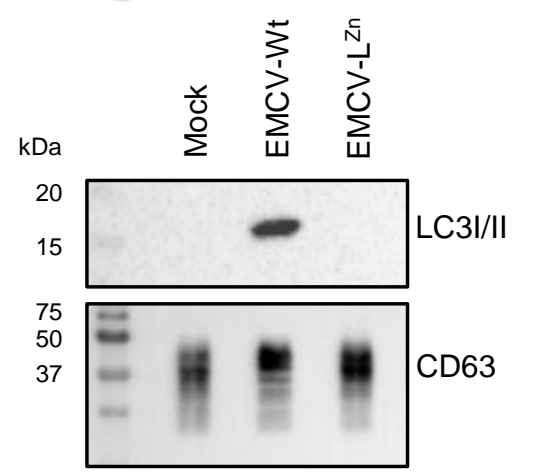

e

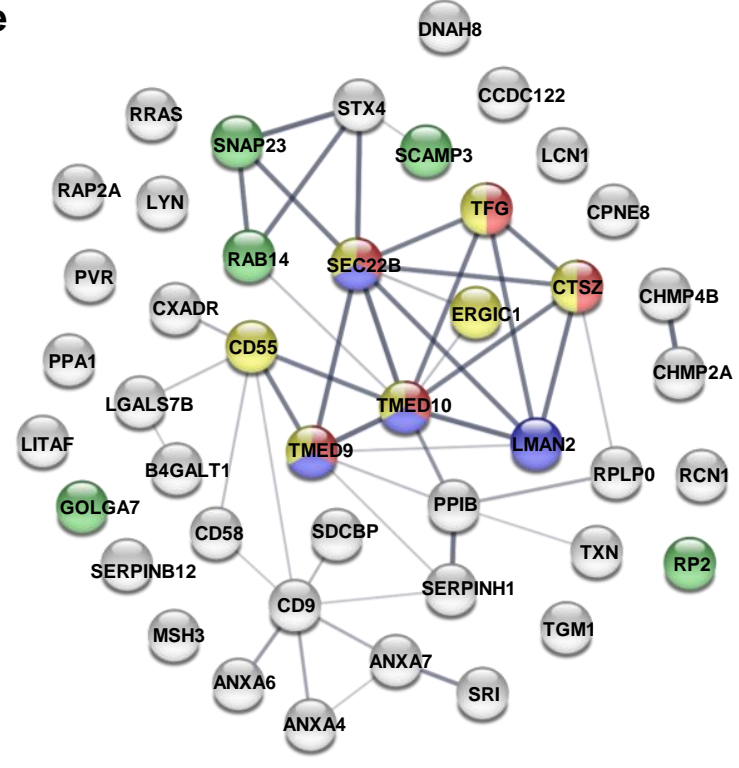

g

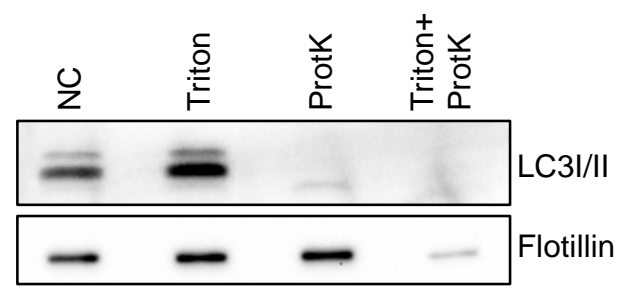




\section{Figure 4}

a

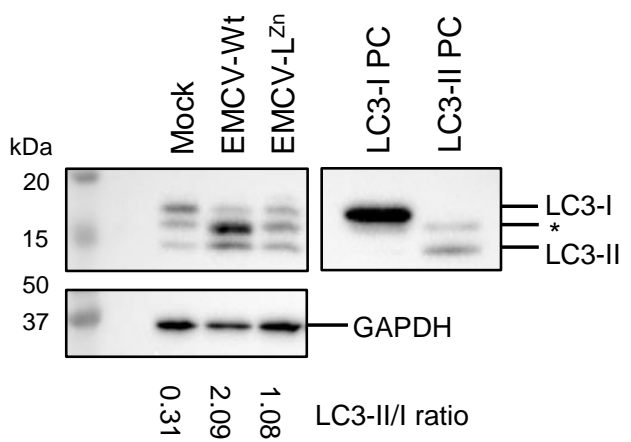

C

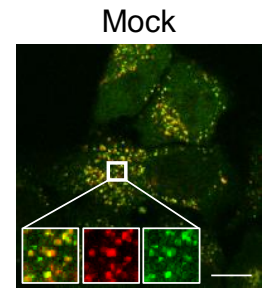

EMCV-Wt
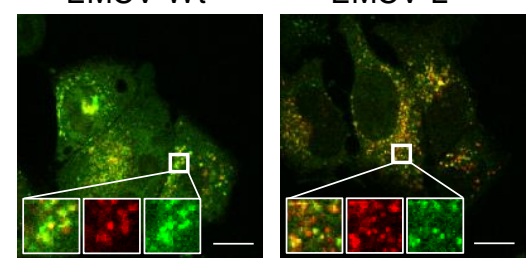

EMCV-LZn

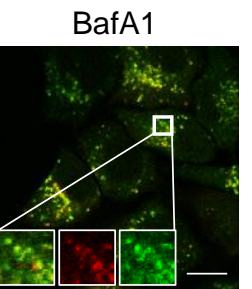

(1) b

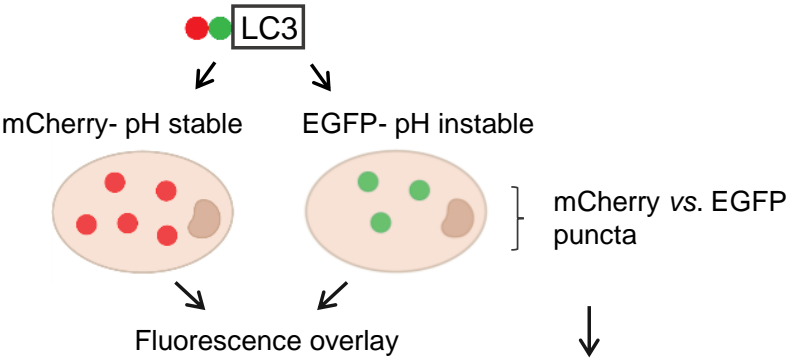

Fluorescence overlay vs. Autolysomes (acidic)
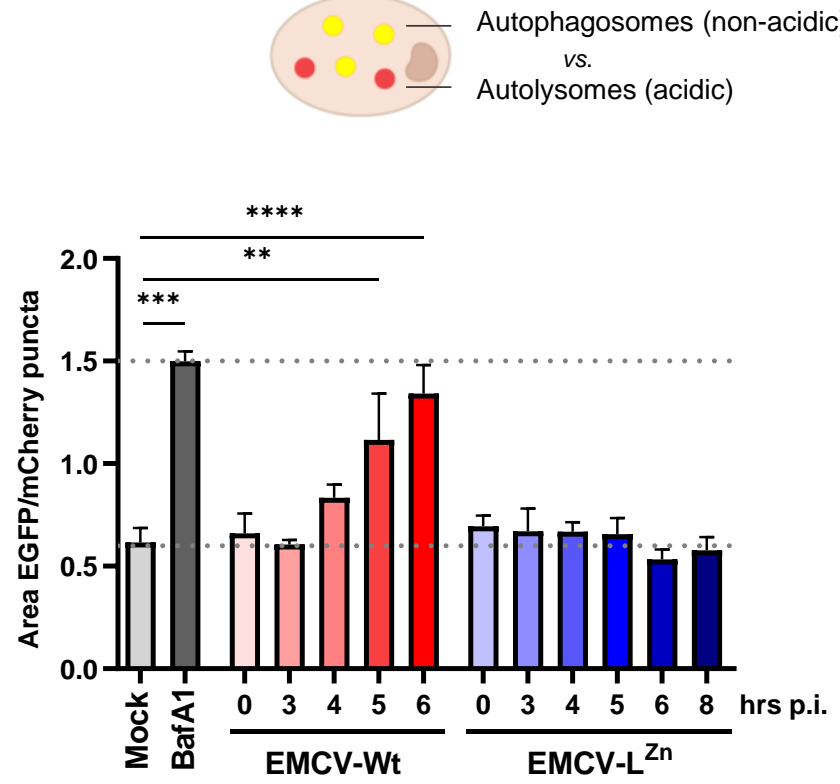

d

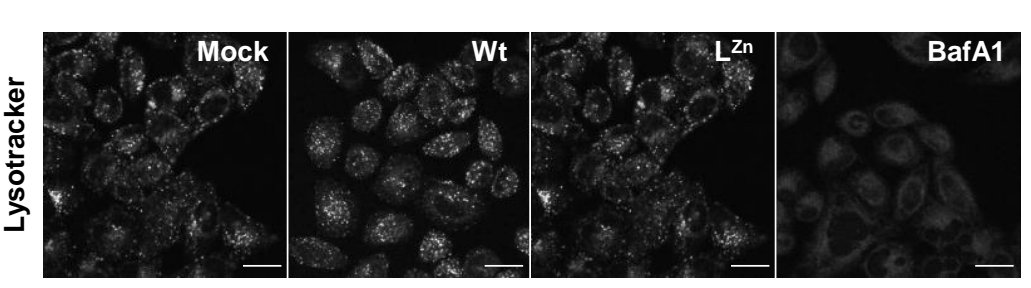

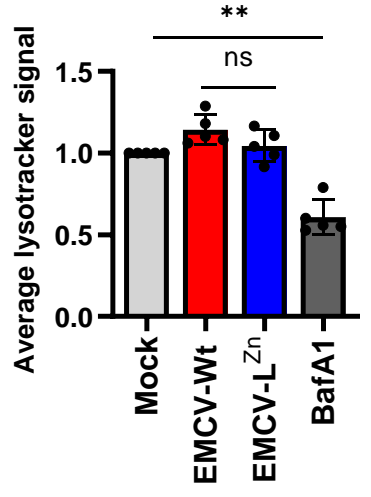




\section{Figure 5}

a

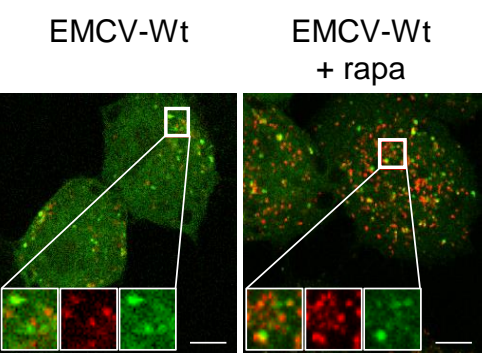

b
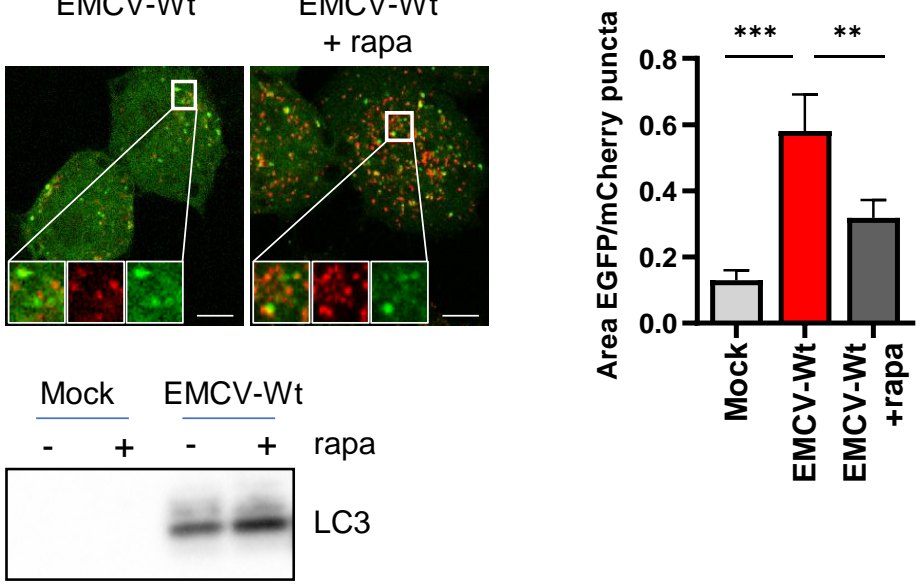

C Total EV-enclosed virus

d $100 \mathrm{~K} \mathrm{EVs}$

$10 \mathrm{~K} \mathrm{EVs}$
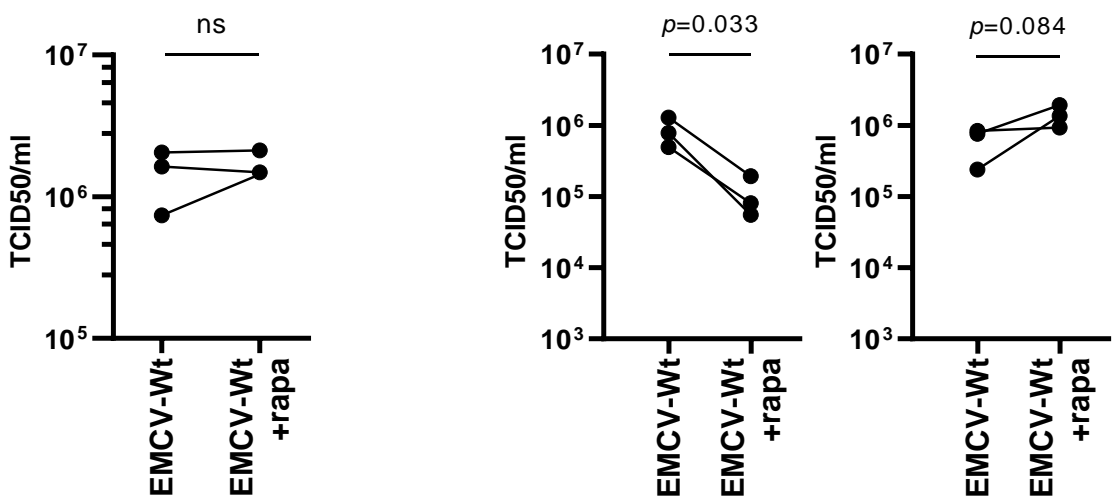


\section{Figure 6}

a

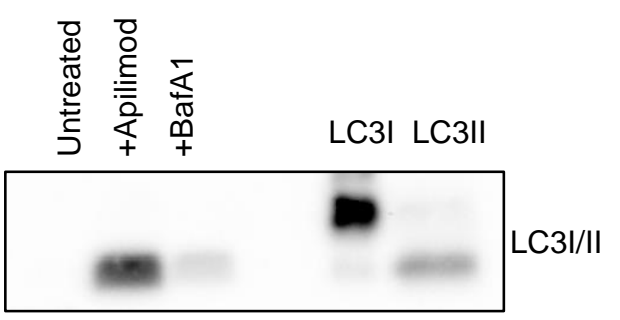

C

EV-enclosed virus

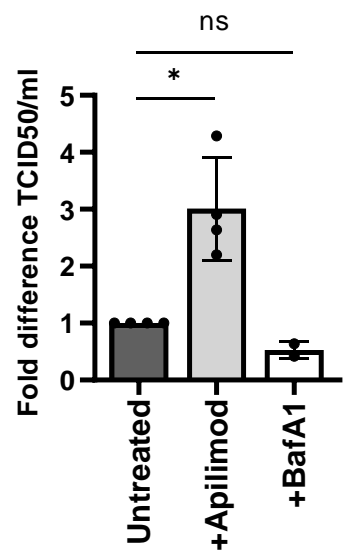

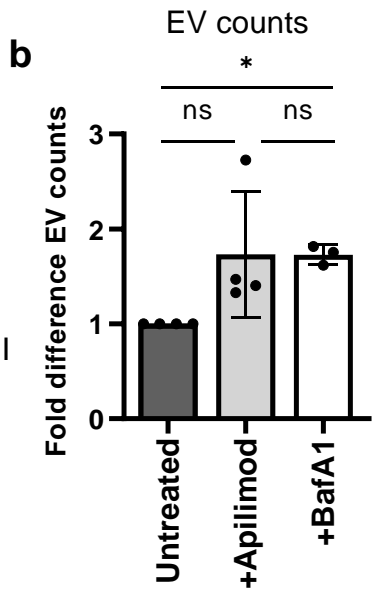

d Intracellular virus

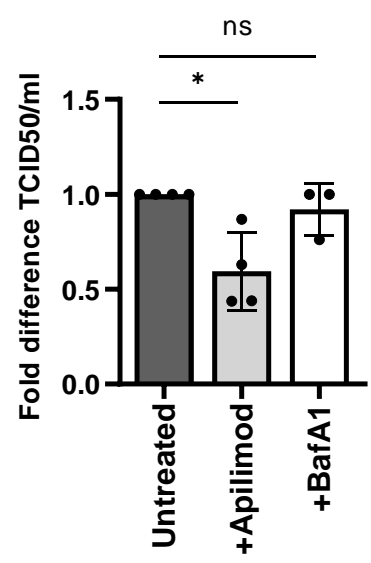

